\title{
Light interactions in flexible conjugated dyes
}

\author{
Jonas Sjöqvist
}

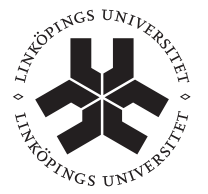

\section{Linköping University} INSTITUTE OF TECHNOLOGY

Linköping University

Department of Physics, Chemistry and Biology

Theory and Modelling

SE-581 83 Linköping, Sweden

Linköping 2014 
(C) Jonas Sjöqvist

ISBN 978-91-7519-282-6

ISSN 0345-7524

Typeset using $\mathrm{LT}_{\mathrm{E}} \mathrm{X}$

Printed by LiU-Tryck, Linköping 2014 


\begin{abstract}
In this thesis methodological developments have been made for the description of flexible conjugated dyes in room temperature spectrum calculations. The methods in question target increased accuracy and efficiency by combining classical molecular dynamics (MD) simulations with time-dependent response theory spectrum calculations.

For absorption and fluorescence spectroscopies a form of conformational averaging is used, where the final spectrum is obtained as an average of spectra calculated for geometries extracted from ground and excited state MD simulations. For infrared and Raman spectroscopies averaged spectra are calculated based on individual spectra, obtained for zero-temperature optimized molecular structures, weighted by conformational statistics from MD trajectories. Statistics for structural properties are also used in both cases to gain additional information about the systems, allowing more efficient utilization of computational resources. As it is essential that the molecular mechanics description of the system is highly accurate for methods of this nature to be effective, high quality force field parameters have been derived, describing the molecules of interest in either the MM3 or CHARMM force fields.

These methods have been employed in the study of three systems. The first is a platinum(II) actylide chromophore used in optical power limiting materials, for which a ultraviolet/visible absorption spectrum has been calculated. The second is a family of molecular probes called luminescent conjugated oligothiophenes (LCOs), used to detect and characterize amyloid proteins, for which both absorption and fluorescence spectra have been calculated. Finally, infrared and Raman spectra have been calculated for a group of branched oligothiophenes used in organic solar cells.

In addition, solvation effects have been studied for conjugated polyeletrolytes in water, resulting in the development of two solvation models suitable for this class of molecules. The first uses a quantum mechanics/molecular mechanics (QM/MM) description, in which the solute molecule is described using accurate quantum mechanical methods while the surrounding water molecules are described using point charges and polarizable point dipoles. The second discards the water entirely and removes the ionic groups of the solute. The QM/MM model provides highly accurate results while the cut-down model gives results of slightly lower quality but at a much reduced computational cost.

Finally, a study of protein-dye interactions has been performed, with the goal of explaining changes in the luminescence properties of the LCO chromophores when in the presence of amyloid proteins. Results were less than conclusive.
\end{abstract}




\section{Populärvetenskaplig sammanfattning}

Kvantmekanik ger en teoretisk grund som med enkla samband hjälper oss beskriva den värld vi lever i. Praktisk sett är det dock inte fullt så enkelt, då det krävs en lång rad förenklingar innan det ens går att utföra beräkningar på några få atomer. De system vi är intresserade av kan innehålla tusentals eller miljontals atomer, så för att kunna studera dem måste vi införa ytterligare approximationer. Ett sätt att göra detta är att blanda klassiska och kvantmekaniska metoder. Då låter man de viktiga delarna av beräkningarna hanteras på kvantmekanisk nivå medan de mindre viktiga, och kanske beräkningsmässigt svåra, delarna beskrivs klassiskt. Den här avhandlingen behandlar utvecklingen av den här sortens metoder med inriktning på uträkningar av olika spektroskopier.

Spektroskopi används för att studera hur ljus växelverkar med olika material och i det här fallet handlar det om hur infrarött, synligt och ultraviolett ljus interagerar med molekyler. De utvecklade metoderna har använts vid studier av tre olika sorters molekyler som används i vitt skilda områden. Den första är framtagen för att agera som skydd för sensorer och är transparent vid låg ljusintensitet men filtrerar bort högintensivt ljus. Den andra sortens molekyler används för att identifiera och studera amyloida proteinansamlingar, vilka är kopplade till ett antal sjukdomar såsom Alzheimers och typ 2-diabetes. Den sista sortens molekyler har strukturella och spektrala egenskaper som gör att de är speciellt lämpade för att användas i organiska solceller.

Vad som är gemensamt för dessa tre uppsättningar molekyler är att de är flexibla, vilket betyder att de ständigt ändrar form vid rumstemperatur, och att deras interaktion med ljus beror starkt på denna form. Detta innebär att det är viktigt att ta i beaktning hur molekylerna rör sig i spektrumberäkningarna. I de utvecklade metoderna görs detta genom att kombinera klassiska dynamiksimuleringar med kvantmekaniskt beräknade spektrum. 


\section{Acknowledgments}

Clearly the most difficult part of any thesis, writing the acknowledgements is fraught with danger. Mention too few and the rest get mad that they weren't included. Mention to many and you dilute whatever value an inclusion had. I hope that I have struck a balance between the two, including only those who truly deserve to be mentioned. So if you've already skimmed these pages and your name hasn't popped out, don't worry, you're probably not forgotten. It's just that I care more about other people than I do about you. I promise that I will at least feel a bit bad about it when I hand over your copy of the thesis.

It's a cliché to start by thanking your supervisor, but some clichés exist for a reason. No one has meant as much for the continued well-being of my academic career as my supervisor Patrick Norman. Whether discussing science, correct English usage or the most aesthetically pleasing placement of a line in a figure, he is always calm, perceptive and, perhaps most importantly, patient. One could not ask for a better supervisor. The only things he has not succeeded in is to get me interested in sports, though I suspect that might be a problem without a solution.

Coming in a close second in importance for my work over the past five years is Mathieu Linares, who acts as a perfect counterbalance to Patrick. He is neither patient nor calm, yet somehow he manages to turn this into a good quality, constantly pushing and encouraging me to become a better scientist. Not only that but he is also a very good friend.

Continuing with scientific collaborators, I wish to thank my co-supervisor Mikael Lindgren, who has been supplying experimental data and insight since my masters thesis. Thanks also to Peter Nilsson and Rozalyn Simon over in the chemistry department, Kurt V. Mikkelsen at the University of Copenhagen, Denmark and María del Carmen Ruiz Delgado at the University of Málaga, Spain.

I would also like to thank everyone at the Laboratory for Chemistry of Novel Materials at the University of Mons, Belgium, with specific mention of David Beljonne and Linjun Wang, for making my stay there a thoroughly pleasant one.

People always say that they couldn't have done it without the support of their friends. Well you won't hear any such hyperbole from me. I'm sure I could have done it without them, but I'm also sure it wouldn't have been nearly as much fun. The following list has been purposefully arranged in alphabetical order, so as not to imply any ranking of the listed people. Because of course you would end up at the top, wouldn't you? 
Bo Durbeej, who always makes sure that I don't think too highly of myself.

Thomas "Dolph" Fransson, for being constantly entertaining.

Cecilia Goyenola, for keeping the feud alive.

Joanna Kauczor, for the cakes, the otters, the emergencies and the friendship.

Paulo V. C. Medeiros, whose skill as a musician kind of makes you want to punch him sometimes, but guiltily, since he's such a nice guy.

Morten Pedersen, for eating my candy, asking me to print stuff and being generally Danish.

Sébastien Villaume, for his strong opinions and his unwillingness to let things go.

Thanks also to everyone else in the computational physics and theoretical physics groups, as well as all the other people who hang around with us, for all the fika breaks, lunches and other adventures.

I would also like to thank the administrative staff, with specific mention of Lejla Kronbäck, without whom we probably wouldn't survive.

While I claim full responsibility for the content of this thesis, including any misspellings, errors or other weirdness, I cannot say the same for its presentation. The reason this thesis looks as good as it does is in large part due to the excellent Latex template created by Olle Hellman, which he graciously supplied me with.

Moving on to people outside of work, I wish to thank my friends Henrik Svensson, Andreas Thomasson and Marcus Wallenberg for the lunches, pool games, organ donations and other fun stuff that we've gotten up to. Erik Tengstrand also deserves thanks for the weekly running sessions, during which both body and mind have been exercised.

And finally, my family.

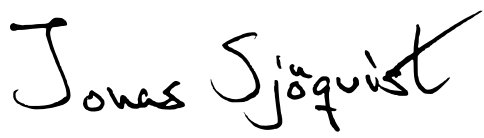

Jonas Sjöqvist

Linköping, August 2014 


\section{CONTENTS}

Notation ix

Acronyms $\mathrm{x}$

1 Introduction 3

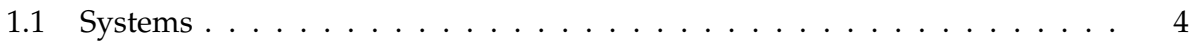

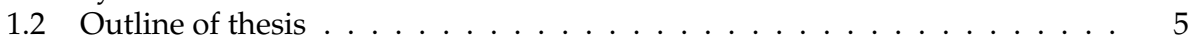

2 Spectroscopy 7

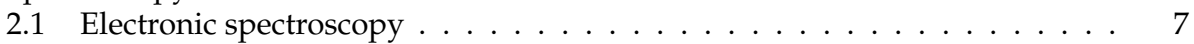

2.2 Vibrational spectroscopy . . . . . . . . . . . . . . . . . . 10

3 Density functional theory 13

3.1 The Born-Oppenheimer approximation . . . . . . . . . . . . . 13

3.2 The Hohenberg-Kohn theorems . . . . . . . . . . . . . . . . . . . . . 15

3.3 The Kohn-Sham equations . . . . . . . . . . . . . . . . . . . . . 16

3.4 The self-consistent field method . . . . . . . . . . . . . . . . . . 17

3.5 Exchange-correlation functionals . . . . . . . . . . . . . . 18

4 Response theory 23

5 Molecular mechanics 29

5.1 Force field terms . . . . . . . . . . . . . . . . . . . . 30

6 Molecular dynamics 35

6.1 Numerical integration . . . . . . . . . . . . . . . . . . . . . 35

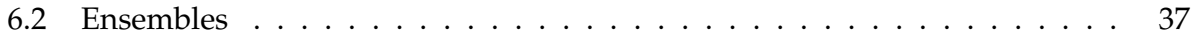

6.3 Geometry optimization . . . . . . . . . . . . . . . . . . 39

7 Solvation models 41

7.1 Quantum mechanics /molecular mechanics . . . . . . . . . . . . . . 42

7.2 Continuum models . . . . . . . . . . . . . . . . . . . . . 44

8 Conformational averaging 47

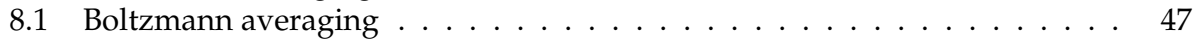

8.2 Molecular dynamics sampling . . . . . . . . . . . . . . . . . 48

9 Protein interaction 51

9.1 Planarization . . . . . . . . . . . . . . . . . . . . 51

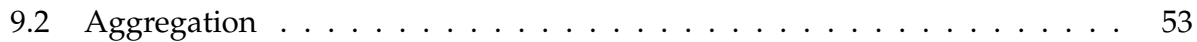

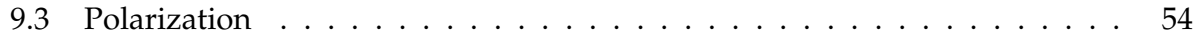

Bibliography 57

List of figures 63 
List of papers and my contributions 66

\section{Paper I 67}

Platinum(II) and phosphorus MM3 force field parametrization for chromophore absorption spectra at room temperature

\section{Paper II 79}

Molecular dynamics effects on luminescence properties of oligothiophene derivatives: A molecular mechanics-response theory study based on the CHARMM force field and density functional theory

\section{Paper III 93}

$Q M / M M-M D$ simulations of conjugated polyelectrolytes: A study of luminescent conjugated oligothiophenes for use as biophysical probes

\section{Paper IV 107}

Towards a molecular understanding of the detection of amyloid proteins with flexible conjugated oligothiophenes

\section{Paper V 125}

A combined MD/QM and experimental exploration of conformational richness in branched oligothiophenes 


\section{NOTATION}

\section{notation}

$a($ ) function

$a$ [ ] functional

$\hat{a}$ operator

a vector

constants

$c$ speed of light in vacuum

$e$ elementary charge

$h$ Planck constant

$\hbar$ reduced Planck constant

$\epsilon_{0}$ vacuum permittivity

$k_{\mathrm{B}}$ Boltzmann constant

coordinates

r electron coordinate

$\mathbf{R}$ atom or nucleus coordinate

v velocity

a acceleration

energies

$E$ total energy

$E_{\mathrm{s}}$ bond stretch energy

$E_{\theta}$ angle bend energy

$E_{\omega}$ torsional energy

$E_{\text {el }}$ electrostatic energy
$E_{\mathrm{vdw}}$ van der Waals energy

$T$ kinetic energy

$V$ potential energy

fields

$F$ electric field

operators

$\hat{H}$ Hamiltonian operator

$\hat{T}$ kinetic energy operator

$\hat{V}$ potential energy operator

$\hat{\mu}$ dipole moment operator

properties

$Z$ atomic number

$m$ atomic mass

$q$ atomic partial charge

$\mu$ dipole moment

$\alpha$ polarizability

$\beta$ first-order hyperpolarizability

$\gamma$ second-order hyperpolarizability

$\epsilon_{r}$ relative permittivity

wave functions

$\Psi$ many-body wave function

$\phi$ single-particle wave function

$n$ electron density 


\section{ACRONYMS}

DFT density functional theory

GGA generalized gradient approximation

IR infrared

LCO luminescent conjugated oligothiophene

LDA local density approximation

MD molecular dynamics

MM molecular mechanics

PCM polarizable continuum model

QM quantum mechanics

QM/MM quantum mechanics/molecular mechanics

UV ultraviolet

UV/Vis ultraviolet/visible 
Based on a true story 



\section{IN T RODUCTION}

It is often said that there are more stars in the sky than grains of sand on all the beaches on Earth, but what is even more astounding is the fact that the number of water molecules in a single glass of water outnumber them both combined. I The world at the atomic level is not only vast on a scale that is hard for the human mind to comprehend, it is also strange, following laws of physics that are not usually encountered in everyday life. The events on this level do matter, however, and in order to study the various action, reactions and interactions that shape the world around us, scientists must investigate this realm of probabilities and dualities. The theoretical framework used to do this is quantum mechanics, the core equations of which are surprisingly simple. The act of actually applying them to a specific problem, on the other hand, is usually anything but simple. At first, only basic test cases could be solved, but with the numerous approximations that have been conceived since then, along with the continually increasing computational power available, the scope has widened from single atoms to molecules to whole systems. But even with these improvements there is still a great need for new models that allow increasingly complex systems to be studied and that improve the accuracy of the calculations performed on them. This thesis deals with the development of just these kinds of models, specifically for the study of spectroscopies.

Spectroscopy is a large field that is in wide use in many areas of research. Based around the interaction of radiated energy with matter, it can reveal a great deal of information for systems ranging from the minuscule to the gargantuan. The light coming from all those stars in the sky reveals the elements that they are composed of and the infrared light that is absorbed when passing through the glass of water can show if there are any contaminants in it. Even your eyes are performing a kind of spectroscopy right now, distinguishing the relative lack of light coming from these letters from the various wavelengths emanating from the white spaces around them.

The methods developed in this thesis deal with the problem of calculating spectra describing the interaction of light with flexible conjugated dyes. The word dye, typically used to mean a substance which gives colour to a material, has a slightly broader definition here, meaning molecules which can be introduced into a system to convey distinct spectral traits. That they are conjugated means that they contain series of alternating single and double bonds, giving them specific spectral properties. These properties are highly sensitive to the geometry of the molecule, meaning that the spectrum can vary significantly for flexible molecules, which move back and forth between various conformations at room temperature. For this reason, there has been a
(I) Back-of-the-envelope calculations puts the number of grains of sand below or near the lower limit for the estimated number of stars in the observable universe, which ranges between $10^{22}$ and $10^{24}$. The number of water molecules in a $250 \mathrm{ml}$ glass of water is roughly $8 \cdot 10^{24}$. 


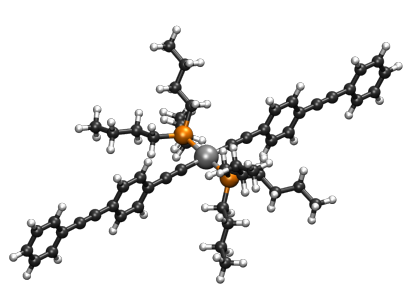

FIGURE 1.1: Platinum(II) acetylide chromophore.

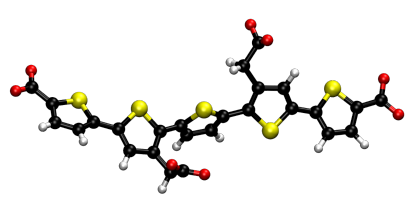

FIGURE 1.2: p-FTAA, one of the luminescent conjugated oligothiophenes.

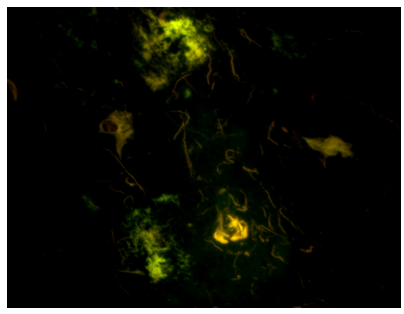

FIGURE 1.3: Fluorescence imaged human Alzheimer's disease tissue section stained by p-FTAA. This image was created by Peter Nilsson and is used with permission. strong focus on the dynamic behaviour of the molecules, and how this affects the calculated spectra, in the developed methods.

\section{Systems}

In Paper I, a platinum(II) acetylide chromophore, ${ }^{1}$ shown in 1.1, was studied using absorption spectroscopy. A chromophore is a molecule or a part of a molecule that absorbs light, and these were developed to protect sensors that detect light, but that can also be damaged by it. An obvious example of such a sensor is the human eye. Shining a high-intensity laser at an eye may not just blind it, but also cause permanent damage. It is not possible to simply filter out certain parts of the incoming light as the lasers can be tuned to the specific wavelengths that the sensors are designed to detect. Instead, it is the increase in intensity that must be detected and protected from. While it is possible to build a physical shield which lowers over the sensor when damaging light is detected, this is a relatively slow process and by the time the shield is in place, enough light has passed through to the sensor to cause damage. The purpose of the studied chromophores is to act as a passive shield, used in addition to an active shield. Materials containing the chromophores are translucent for low light intensities, allowing light to pass through to the sensor, but become opaque for higher intensities, absorbing the incoming light. This allows it to sit in front of the sensor during normal operation, letting light pass through, but immediately responding by absorbing the light when it reaches damaging levels. The material is only capable of doing this for a short time before it becomes saturated, but it is long enough for the active shield to be put in place, offering a more permanent protection.

Papers II, III and IV use absorption and fluorescence spectroscopy to study a set of chromophores known as luminescent conjugated oligothiophenes (LCOs), ${ }^{2,3,4}$ used in the study of amyloid diseases such as Alzheimer's disease and type II diabetes. Amyloid diseases are characterized by the misfolding of naturally occurring proteins in the body. These misfolded proteins stack and form fibrils, which themselves aggregate and create large bundles known as amyloids. Much is still unknown about the exact pathology of these diseases and how they relate to the formation of amyloids, and because of this there is a great deal of interest in the study of protein aggregation. The conventional way of doing this is to stain tissue samples with dyes such as thioflavin $\mathrm{T}^{5}$ and Congo red, ${ }^{6}$ which change the way they emit light when bound to amyloid proteins, allowing them to be detected. These dyes are very good at showing whether there are amyloids in a sample or not, and if so, where they are, but they reveal little about the underlying structure of the aggregates. The LCOs, on the other hand, are sensitive also to this aspect of the 
proteins, absorbing and emitting light at different wavelengths depending on the structure of the protein to which they are bound. Figure 1.2 shows p-FTAA, one of the LCOs, and Figure 1.3 shows a fluorescence image in which it has been used to stain amyloid aggregates in an Alzheimer's disease tissue section, with different colours identifying different amyloid structures.

Finally in Paper V, a set of branched oligothiophenes, 7,8 an example of which is shown in Figure 1.4, has been studied using infrared and Raman spectroscopy. These molecules are intended for use in organic solar cells, which convert the energy of light into electrical current. Organic solar cells consist of two layers of materials, known as the donor and acceptor layers. Light is absorbed by the donor material, which causes a transfer of electrons to the acceptor, from which they are transported and used as current. In order to maximize the efficiency of the solar cells, both the spectral and structural properties of these materials must be tuned to make this process as easy as possible. The spectral properties to ensure that light is absorbed and electrons separated, and the structural properties to maximize the contact surface between the two layers. The branched oligothiophenes are interesting as donor materials because of the disordered structures that they form, generating a large contact area with the acceptor layer.

\section{Outline of thesis}

Chapter 2 gives an introduction to the spectroscopies studied in this work while Chapters 3 to 8 contain the relevant theory and methods, heavily revised and expanded from my licentiate thesis. ${ }^{9}$ Chapter 9 reports some unpublished work concerning the interaction of LCOs with amyloid proteins. Finally, the papers, along with any supporting information, are included.

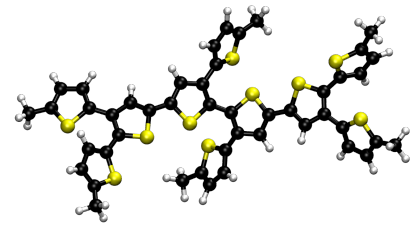

FIGURE 1.4: 6TB, one of the branched oligothiophenes. 



\section{SPECTROSCOPY}

The field of spectroscopy is large and varied, with ties to many natural phenomena, but the basic principle behind all of them can be succinctly summarized as the interaction of matter and radiated energy. What type of energy and how it interacts with matter determines the kind of spectroscopy. The energy most commonly studied is in the form of electromagnetic waves, but other forms include particles, such as electrons and neutrons, as well as acoustic waves. The type of interaction further divides the spectroscopies into categories such as absorption, emission and scattering, with further divisions based on the energy region of the interacting waves and how that energy affects the material.

This work deals with four types of spectroscopies, all concerning the interaction of molecules with electromagnetic radiation, which can either be seen as a wave or as a particle, a photon. The electromagnetic spectrum, shown in Figure 2.1, ranges from low to high energy or, equivalently, from long to short wavelength, with their relationship in vacuum given by

$$
E=\frac{h c}{\lambda},
$$

where $E$ is the energy of the wave, $h$ is the Planck constant, $c$ is the speed of light and $\lambda$ is the wavelength. The type of electromagnetic radiation ranges from low energy radio waves, through the short span of wavelengths visible to the human eye and up to high energy gamma rays, caused by the decay of atomic nuclei. The spectroscopies studied in this work deal with radiation either in the ultraviolet/visible (UV/Vis) or in the infrared (IR) region. UV/Vis radiation is capable of causing excitations into low energy electronic states, and as such is used in electronic spectroscopies. The lower energy IR radiation, on the other hand, interacts with the vibrational motion of the molecule and is used in vibrational spectroscopies. The two electronic spectroscopies, UV/Vis absorption and fluorescence, and the two vibrational spectroscopies, IR absorption and Raman scattering, are detailed in the following sections.

\section{Electronic spectroscopy}

\section{UV/VIS ABSORPTION}

For an atom or molecule, there exists a discrete set of allowed electronic states, each of which can be interpreted as representing a possible spatial distribution, or rather probability distribution, of the electrons in the system. Each state has an associated energy and assuming no outside influence, the system is found in that

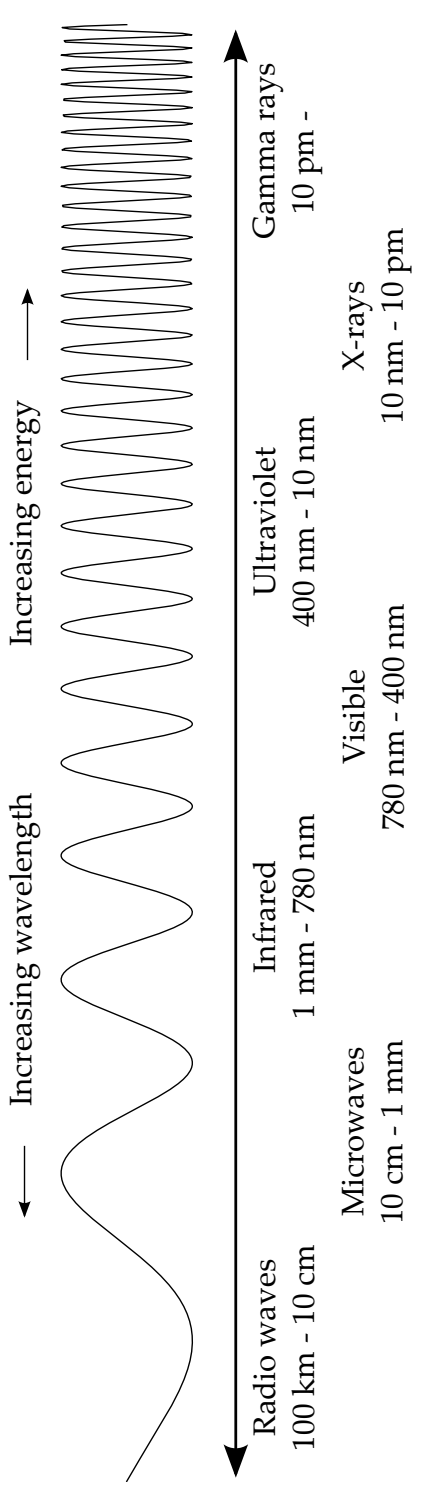

FIGURE 2.1: The electromagnetic spectrum with approximate spectral regions. 
which has the lowest possible energy, the ground state. Transitions between states can only occur if energy matching the difference between the current state and one of the others is added or subtracted from the system. One way that excitation can occur is when incident electromagnetic radiation oscillates with a wavelength that corresponds to an allowed transition energy. Upon absorption, the energy of the radiation is expended in promoting the system into an excited state. ${ }^{10,11}$

The wavelength corresponding to the first possible electronic transition, between the ground state and the first excited state, typically falls within the visible or ultraviolet (UV) regions of the spectrum, which are the regions studied in this thesis. Such excitations can be interpreted as mostly affecting the valence electrons of the system, as the redistribution of the electrons that occurs in the excited state is primarily located in the areas further away from the nuclei. Higher energy radiation, such as x-rays, on the other hand, are capable of exciting core electrons and even causing ionization, separating an electron from the system entirely.

Excitations that occur within the visible spectrum can be observed by the human eye, such as for chlorophyll, which absorbs light in the red and blue regions, ${ }^{12}$ leaving the green light which gives plants their colour. For hemoglobin, which transports oxygen in blood, the states of the molecule are altered as oxygen is bound to it, causing the absorption of red light to decrease, ${ }^{13}$ making oxygenated blood appear a deeper red compared to deoxygenated blood. Not only does this show that small changes in the molecule can noticeably alter its spectral properties, but such changes can also be very useful, as it is possible to measure the oxygen content of blood from its absorption spectrum.

Depending on the type of absorption, there are several factors which influence the probability of it occurring. In this work, linear absorption is studied, which means that a single photon is absorbed and that the strength of this absorption depends linearly on the amplitude of the corresponding electric field. There are also weaker, non-linear processes which can occur, such as two- or three-photon absorption, where the energies of several photons add up to the excitation energy and are absorbed simultaneously. The strength of an excitation also depends on the absorbed energy and the transition dipole moment between the initial and final state. This dependence on how the transition dipole moment changes can be used to make predictions regarding the strength of a specific absorption based only on the symmetry of the two states between which the excitation occurs.

While the possible electronic excitations form a discrete set, each represented by a single excitation energy, the absorption always occurs for a continuous range surrounding this specific point. Such spectral broadening can have a number of causes, ${ }^{11,14}$ a few of which will be listed here. Natural broadening is the most 
basic kind, also known as Heisenberg broadening due to the fact that its origin relates to the Heisenberg uncertainty principle. As the product of the uncertainty in energy and the uncertainty in time has to be larger than $\hbar / 2$, the absorbed energy varies slightly depending on the excitation lifetime. This effect is small, however, with a more significant one due to Doppler broadening, seen most clearly for systems in the gas phase. Depending on the temperature of the system, the absorbing molecules will be moving to some degree with respect to the source of the electromagnetic radiation. This will cause a slight blue- or redshift of the radiation as seen from the perspective of the molecule, meaning that there is a possibility that it will be absorbed even if it does not match the excitation energy exactly. Finally, there is vibrational and rotational broadening, caused by the fact that excitations can occur from a number of vibrational and rotational states in the original electronic state into another set of vibrational and rotational states for the final electronic state. These sources of broadening stack on top of each other, as for each vibrational state there is a set of rotational levels, which are further broadened by Doppler and natural broadening. An example of this is shown in Figure 2.2.

\section{FLUORESCENCE}

This thesis deals with photoluminescence, the emission of light by an atom or molecule that has previously been promoted into an excited state by absorption of electromagnetic radiation. ${ }^{10,15}$ There are two kinds of luminescence, the first of which has been studied in this work. They are: fluorescence, where the de-excitation occurs from a singlet state and phosphorescence, where it occurs from a triplet state. While the fluorescence process is relatively fast, with emission following absorption by only a few nanoseconds, phosphorescence may be a very slow process, with emission occurring up to several hours after the initial absorption.

The same rules that govern absorption also apply to fluorescence, meaning that the emitted radiation will correspond to the difference in energy between the excited state and the ground state and that the probability of emission will depend on that energy as well as the transition dipole moment between the two states, with broadening occurring for the same reasons. The fluorescence spectrum is not generally the same as the absorption spectrum, however, usually displaying a redshifted and mirrored profile. This is due to the fact that absorption often occurs to higher vibrational states of the excited electronic state. During the time that the molecule spends in the excited state, the vibrational energy is dissipated to the environment and a relaxation occurs to a lower vibrational state. When emission finally happens, it may be to a higher vibrational state, resulting in an

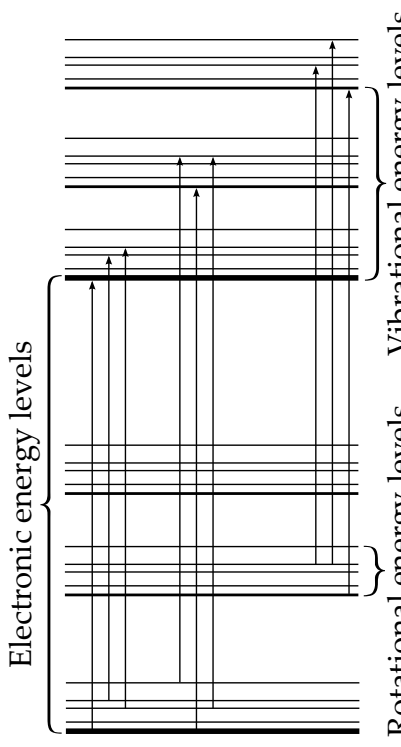

FIGURE 2.2: Excitations between the same electronic states, but different vibrational and rotational states. 

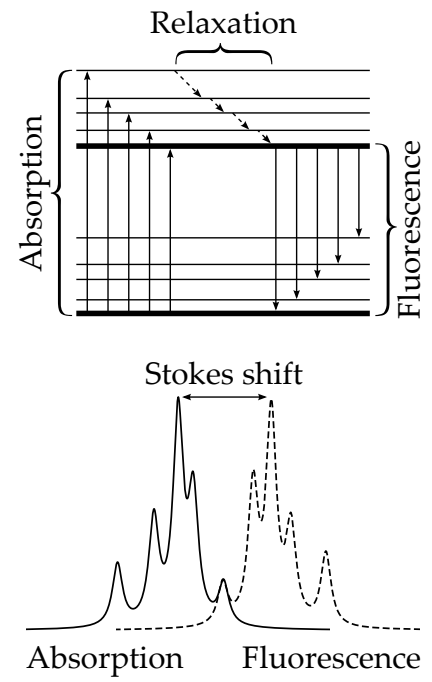

FIGURE 2.3: Illustration of the processes leading to a redshift of the fluorescence spectrum and the resulting Stokes shift. emitted photon of lower energy than the one that was absorbed. The mirroring of the absorption and emission spectra comes from the fact that both absorption and emission generally occur from the vibrational ground states of the electronic ground and excited states, respectively. Thus, the absorption spectrum shows the vibrational levels of the electronically excited state while the emission spectrum does the same for the ground state. The difference between the peak of the absorption spectrum and that of the fluorescence spectrum is know as the Stokes shift. Figure 2.3 illustrates this process.

An interesting practical example of this can be found in laundry detergents. These often contain dyes called optical brighteners, ${ }^{16}$ which absorb light in the UV region but emit it in the visible range. This means that a piece of clothing that has been washed with the detergent is actually capable of emitting more visible light than is shone on it, making it appear brighter and cleaner. This effect is further enhanced by the fact that the emitted light is in the blue region, which counteracts the generally yellow appearance of fabric contaminants.

\section{Vibrational spectroscopy}

\section{IR ABSORPTION}

Absorption can still occur in a molecule even if the incident radiation does not have the energy to achieve an electronic excitation, instead causing vibrations of the nuclei. ${ }^{10,11,17}$ This type of molecular motion is excited by electromagnetic radiation in the IR region and may be very useful in the identification of chemical compounds. The vibrations are often localized to specific functional groups or structural features of the molecule, which produce characteristic peaks in the spectrum. Based on this, an inventory of structural features can be created from which the structure of the molecule can be deduced. It is also possible to use IR spectroscopy to determine the concentration of a certain kind of molecule in a sample, as is done for some types of breathalysers, ${ }^{18}$ which determine the alcohol content in breath samples. This is done by measuring the absorption band characteristic for oxygen-hydrogen bond vibrations and comparing it to calibration values.

In the Born-Oppenheimer approximation, where the motion of the nuclei and electrons are decoupled, the nuclei can be seen as moving on the potential energy surface created by the electrons. By studying the shape of this potential energy surface near minima it is possible to find a discrete set of fundamental vibrations, known as normal modes, each capable of vibrating independently of the others. For each mode, there is a set of allowed vibrational levels which are based on the curvature of the potential energy surface around the minimum. When com- 
puting the energy levels, a common approximation is to assume that all vibrational modes act as independent harmonic oscillators, which gives an equal difference in energy between each successive level. While this is a reasonable approximation for the lower levels, in reality the energy gap becomes lower for each new level, eventually leading to bond dissociation. The vibrational state of the system as a whole is defined by stating the population of the vibrational states for each mode. Absorption can occur when the frequency of the electromagnetic radiation matches that of the energy difference between an occupied and unoccupied vibrational state for one of the modes. The strength of such an absorption depends on the transition dipole moment between the two vibrational states, but is often approximated as being dependent on the change in the dipole moment of the molecule upon vibration. ${ }^{10}$ This is a useful approximation as it makes it possible to intuitively gauge the strength of absorption based on the manner in which the atoms vibrate.

A non-linear molecule containing $N$ atoms has $3 N$ degrees of freedom but only $3 N-6$ normal modes. The remaining six degrees of freedom represent translational motion in three directions and rotational motion around each of the axes. In the linear case, one rotational degree of freedom disappears, leaving $3 N-5$ vibrational modes. These modes can be divided into categories depending on the type of vibration they represent, with stretching, bending and twisting being three examples. Stretching modes generally have higher frequencies than bending modes, which in turn have higher frequencies than twisting modes. Using the standard example of water, there are three atoms, nine degrees of freedom and three vibrational modes. The modes, shown in Figure 2.4, are categorized as bending, symmetric stretching and asymmetric stretching. As shown in the calculated IR spectrum of Figure 2.5, the two stretching modes are quite close in energy while the bending mode is significantly lower. It can also be seen that the bending mode has the strongest absorption while the symmetric stretching mode is much weaker, with the asymmetric stretching mode ending up in between. This can be intuitively understood by considering how the dipole moment of the molecules changes with the vibrations. The bending mode has a large effect on the size of the dipole moment but does not change its direction, giving it a strong absorption. For the asymmetric stretching mode the situation is reversed, with large changes in the direction of the dipole moment, but only small changes in its size, also resulting in strong absorption. The symmetric stretching mode, on the other hand, neither changes the direction of the dipole moment nor alters its size to any significant degree, resulting in the weakest absorption. Such reasoning based on simple symmetry arguments can provide an easy way of identifying forbidden transitions and estimating relative intensities.

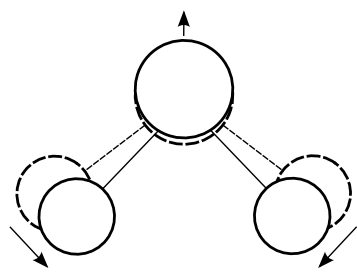

Bending

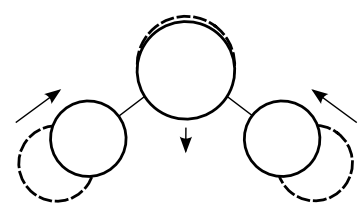

Symmetric stretching

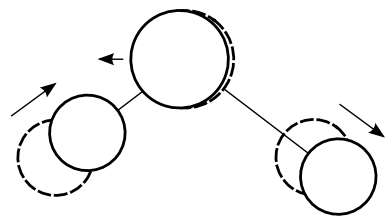

Asymmetric stretching

FIGURE 2.4: The vibrational modes of water.

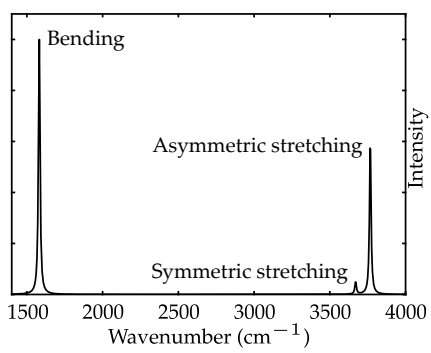

FIGURE 2.5: IR spectrum of water, calculated at the B3LYP/ccpVTZ level of theory. The energy scale uses wavenumbers, the traditional energy unit in vibrational spectroscopy. 


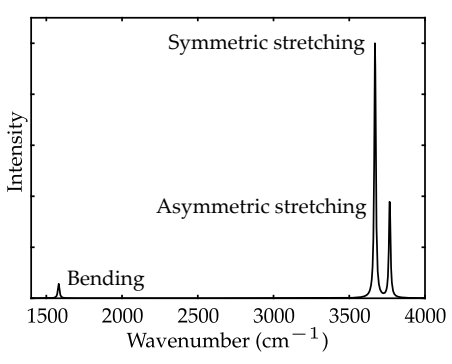

FIGURE 2.6: Raman spectrum of water, calculated at the B3LYP/cc-pVTZ level of theory.

\section{RAMAN SCATTERING}

In Raman scattering, the vibrational state of the system is altered, same as for IR absorption, but the process by which this occurs is different. ${ }^{10,11,17}$ Scattering is a two-photon process in which a photon which does not match any of the allowed transitions in the system causes a short-lived excitation into a virtual state. This is immediately followed by the emission of a photon, returning the system to one of the allowed states. If the initial and final states are the same, this is known as Rayleigh scattering, which is an elastic scattering, altering only the direction of the photon. If the two states are different, however, the energy of the scattered photon will have changed. This is called Raman scattering, with Stokes and anti-Stokes variants depending on if the scattered photon has been red- or blueshifted, respectively. Both Rayleigh and Raman scattering are unlikely processes, with Raman scattering being the less probable of the two by a factor of roughly one thousand. ${ }^{17}$

While the intensity of IR absorption could be approximated as being proportional to the change in dipole moment upon vibration, the same approximation in Raman scattering leads to a dependence on the change in polarizability. This is a much harder property to estimate intuitively, but usually the IR and Raman spectra are complementary, i.e. modes that are weak in IR are often strong in Raman and vice versa. This can be seen in the calculated Raman spectrum of water, shown in Figure 2.6, where the symmetric stretching mode is now the strongest while the bending mode is weak. By combining IR and Raman spectroscopy, it is possible to find most of the vibrational modes, giving a more complete picture of the studied molecule. 


\section{DENSITY FUNCTIONAL THEORY}

Over the 19th century, experimental evidence had been mounting suggesting that there was something fundamental in the field of physics that was not fully understood. Experiments concerning black-body radiation and the photoelectric effect could not be explained by the currently available theories, but at the start of the 20th century, a number of important theoretical discoveries were made which helped explain these phenomena. These include Planck's law of black-body radiation ${ }^{19}$ and Einstein's explanation of the photoelectric effect in $1905,{ }^{20}$ in which light is described as being composed of discrete quanta, i.e. photons. This started the field of quantum mechanics, leading Schrödinger to formulate a description of matter in the form of waves, resulting in the equation that bears his name. ${ }^{21}$

The non-relativistic, time-independent Schrödinger equation has the following form:

$$
\hat{H} \Psi=E \Psi,
$$

where $\Psi$ is the many-body wave function describing both the nuclei and electrons of the system and $E$ is its total energy. The Hamiltonian operator, $\hat{H}$, unaffected by any external potential, can be written as

$$
\begin{aligned}
\hat{H}= & \hat{T}_{n}+\hat{T}_{e}+\hat{V}_{n n}+\hat{V}_{e e}+\hat{V}_{n e} \\
= & -\sum_{k} \frac{\hbar^{2}}{2 m_{k}} \nabla_{k}^{2}-\sum_{i} \frac{\hbar^{2}}{2 m_{e}} \nabla_{i}^{2}+\sum_{i<j} \frac{e^{2}}{4 \pi \epsilon_{0}\left|\mathbf{r}_{i}-\mathbf{r}_{j}\right|} \\
& -\sum_{i, k} \frac{e^{2} Z_{k}}{4 \pi \epsilon_{0}\left|\mathbf{r}_{i}-\mathbf{R}_{k}\right|}+\sum_{k<l} \frac{e^{2} Z_{k} Z_{l}}{4 \pi \epsilon_{0}\left|\mathbf{R}_{k}-\mathbf{R}_{l}\right|},
\end{aligned}
$$

where $\hat{T}_{n}$ and $\hat{T}_{e}$ are the kinetic energy operators for the nuclei and electrons, respectively, and $\hat{V}_{n n}, \hat{V}_{e e}$ and $\hat{V}_{n e}$ are potential energy operators that give the interaction energy among nuclei, electrons and between the two, respectively.

While seemingly simple, the Schrödinger equation can only be solved analytically for the most basic of systems. For it to be put to any practical use studying real systems, approximations must be made. This chapter will follow one possible approximation path, density functional theory (DFT), which makes it possible to study systems containing hundreds of atoms. It starts, like most electronic structure methods, with the Born-Oppenheimer approximation.

\section{The Born-Oppenheimer approximation}

The first step of the Born-Oppenheimer approximation ${ }^{22}$ is the ansatz that the wave function of the system can be divided into 
nuclear and electronic components as

$$
\Psi=\Psi_{e}(\mathbf{R}, \mathbf{r}) \Psi_{n}(\mathbf{R}),
$$

where the nuclear wave function depends only on the nuclear coordinates while the electronic wave function depends on both nuclear and electronic coordinates. It is also assumed that the electronic wave function is constructed in such a way as to satisfy the Schrödinger equation for electrons in the presence of static nuclei, which has the Hamiltonian

$$
\begin{aligned}
\hat{H}_{e} & =\hat{T}+\hat{V}_{e e}+\hat{V}_{n e} \\
& =-\sum_{i} \frac{\hbar^{2}}{2 m_{e}} \nabla_{i}^{2}+\sum_{i<j} \frac{e^{2}}{4 \pi \epsilon_{0}\left|\mathbf{r}_{i}-\mathbf{r}_{j}\right|}-\sum_{i, k} \frac{e^{2} Z_{k}}{4 \pi \epsilon_{0}\left|\mathbf{r}_{i}-\mathbf{R}_{k}\right|}
\end{aligned}
$$

and the eigenvalues

$$
\hat{H}_{e} \Psi_{e}(\mathbf{R}, \mathbf{r})=\varepsilon_{e} \Psi_{e}(\mathbf{R}, \mathbf{r}),
$$

where $\varepsilon_{e}$ depends parametrically on $\mathbf{R}$. The full Hamiltonian acting on the full wave function can then be written as

$$
\begin{aligned}
\hat{H} & \Psi(\mathbf{R}, \mathbf{r}) \\
= & \left(\hat{T}_{n}+\hat{V}_{n n}+\hat{H}_{e}\right) \Psi_{e}(\mathbf{R}, \mathbf{r}) \Psi_{n}(\mathbf{R}) \\
= & \Psi_{e}\left[-\sum_{k} \frac{\hbar^{2}}{2 m_{k}} \nabla_{k}^{2}+\sum_{k, l} \frac{e^{2} Z_{k} Z_{l}}{4 \pi \epsilon_{0}\left|\mathbf{R}_{k}-\mathbf{R}_{l}\right|}+\varepsilon_{e}\right] \Psi_{n}(\mathbf{R}) \\
& -\sum_{k} \frac{\hbar^{2}}{2 m_{k}}\left(2 \nabla_{k} \Psi_{n}(\mathbf{R}) \nabla_{k} \Psi_{e}(\mathbf{R}, \mathbf{r})+\Psi_{n}(\mathbf{R}) \nabla_{k}^{2} \Psi_{e}(\mathbf{R}, \mathbf{r})\right) .
\end{aligned}
$$

The energy $\varepsilon_{e}$ can here be identified as the adiabatic contribution of the electrons to the total energy of the system, i.e. the energy of electrons that respond instantly to changes in the nuclear coordinates. The second term in Equation 3.6 is the nonadiabatic contribution to the energy, as it contains a dependence on $\nabla_{k} \Psi_{e}(\mathbf{R}, \mathbf{r})$, where $k$ is one of the nuclei.

At this point it is observed that the even the smallest nucleus, the single proton of a hydrogen atom, is over 1800 times heavier than an electron. The Coulomb forces experienced by the particles are in the same order of magnitude, however, making it reasonable to assume that the nuclei of the system will be moving at speeds far lower than those of the electrons. Based on this information, the adiabatic approximation can be made, i.e. that the electrons are moving fast enough that they can be seen as responding instantly to any movement of the slow nuclei. In this approximation the contribution of the second term in Equation 3.6 becomes zero, leaving only the contribution of the adiabatic electrons. 
This means that the problem can be split into two parts. First, the electronic wave function is found for stationary nuclei using the electronic Hamiltonian in Equation 3.4. The wave function for the nuclei can then be found from

$$
\hat{H}_{n} \Psi_{n}(\mathbf{R})=\left(\hat{T}_{n}+\hat{V}_{n n}+\varepsilon_{e}\right) \Psi_{n}(\mathbf{R}),
$$

where the solution of the electronic wave function enters as a potential energy surface on which the nuclei move. This way, the Schrödinger equation has been reduced into two simpler problems. This is a large step in the right direction and has reduced the complexity of the problem greatly, but several more steps are needed. Even with this simplified formulation, it is still impossible to find solutions for anything but the simplest of systems.

\section{The Hohenberg-Kohn theorems}

There are a large number of approaches to further reduce the complexity of the electronic structure problem, such as HartreeFock and related post-Hartree-Fock methods, but in this work all such calculations have been performed using DFT. In DFT, the electron density, $n(\mathbf{r})$, is used to describe the system instead of the wave function and the following section details how this is achieved. While a proper derivation should take spin into account, this causes the expressions to become somewhat cluttered, obscuring the ideas behind them. For this reason, spin has been left out of this discussion, focusing instead on the main ideas of DFT. For a more thorough derivation, see e.g. the work of Jacob and Reiher. ${ }^{23}$ Keeping this in mind, the electron density is given by

$$
n\left(\mathbf{r}_{1}\right)=N \int \cdots \int\left|\Psi_{e}\left(\mathbf{r}_{1}, \mathbf{r}_{2}, \cdots \mathbf{r}_{N}\right)\right|^{2} d \mathbf{r}_{1}, d \mathbf{r}_{2} \cdots d \mathbf{r}_{N},
$$

which reduces the degrees of freedom from $3 N$ for the $N$ electrons of the wave function to just 3 for the electron density. While this is clearly a much simpler description, it is not immediately apparent that it gives a full description of the system. However, in 1964, Hohenberg and Kohn showed that an electron density description was fully equivalent to a wave function description and that it could be used to find the ground state of the system. This was summarized in the two Hohenberg-Kohn theorems, ${ }^{24}$ the first of which states that the external potential, $V_{\text {ext }}$, is, up to a constant potential, uniquely defined by the ground state electron density, $n_{0}$. This, together with the fact that the number of electrons of the system can be obtained by integrating the electron density over all space as

$$
N=\int n(\mathbf{r}) d \mathbf{r}
$$


means that the Hamiltonian can be fully reconstructed from just the electron density, and with it the opportunity to find the wave function. The electron density, though seemingly much less complex than the wave function, contains exactly the same information.

The second theorem states that there exists an energy functional, $E[n]$, which, for a given external potential, $V_{\text {ext }}$, has as its minimum the exact ground state energy and that this energy is obtained for the ground state density, $n_{0}$. Using the variational principle, it is then possible to find the ground state density by minimizing $E[n]$ with respect to $n$, and under the constraint that it is possible to derive the density from an $N$-electron antisymmetric wave function. The energy functional can be written as

$$
\begin{aligned}
E[n] & =T[n]+V_{e e}[n]+\int n(\mathbf{r}) V_{\mathrm{ext}}(\mathbf{r}) d \mathbf{r} \\
& =F[n]+\int n(\mathbf{r}) V_{\mathrm{ext}}(\mathbf{r}) d \mathbf{r},
\end{aligned}
$$

where the kinetic energy and Coulomb interaction of the electrons have been combined into the functional $F[n]$. As this functional does not depend on $V_{\text {ext }}$ in any way it is completely system independent. The main obstacle at this point is that the true form of the universal $F[n]$ functional is not known, and without it there is little that can be done to find the ground state density.

\section{The Kohn-Sham equations}

The way around this problem was proposed a year later, in 1965, by Kohn and Sham. ${ }^{25}$ In the Kohn-Sham ansatz, the fully interacting many-body system is replaced by an easier to solve auxiliary system containing non-interacting electrons and which is constructed in such a way as to have the same ground state electron density as the real system. The universal functional can be written as

$$
F[n]=T_{s}[n]+J[n]+E_{\mathrm{xc}}[n],
$$

where $T_{s}[n]$ is the kinetic energy of the non-interacting electrons and $J[n]$ is the Coulomb interaction of the electron density with itself. The term $E_{\mathrm{xc}}[n]$ is known as the exchange-correlation energy which, when the definition of $F[n]$ in Equation 3.10 is taken into account, must be equal to

$$
E_{\mathrm{xc}}[n]=T[n]-T_{s}[n]+V_{e e}[n]-J[n] .
$$

The exchange-correlation energy thus contains the differences in kinetic and interaction energy of the electrons in the real system and the non-interacting auxiliary system. 
Applying the variational principle gives

$$
\begin{aligned}
\frac{\delta E}{\delta n} & =\frac{\delta}{\delta n}\left(T_{s}[n]+J[n]+E_{\mathrm{xc}}[n]+\int n(\mathbf{r}) V_{\mathrm{ext}}(\mathbf{r}) d \mathbf{r}\right) \\
& =\frac{\delta T_{s}[n]}{\delta n}+\frac{\delta J[n]}{\delta n}+\frac{\delta E_{\mathrm{xc}}[n]}{\delta n}+V_{\mathrm{ext}}(\mathbf{r}) \\
& =\frac{\delta T_{s}[n]}{\delta n}+V_{\mathrm{eff}}=\mu,
\end{aligned}
$$

where $\mu$ is a Lagrange multiplier that appears due to the constraint that the electron density must integrate to $N$. With the introduction of the potential $V_{\text {eff }}$, this can be interpreted as the energy minimization of a system of non-interacting particles, moving in an effective potential. Given that the potential has the form

$$
V_{\text {eff }}=\frac{\delta J[n]}{\delta n}+\frac{\delta E_{\mathrm{xc}}[n]}{\delta n}+V_{\mathrm{ext}}(\mathbf{r}),
$$

the ground state electron density found as the solution to the non-interacting system is exactly that of the interacting system. The Hamiltonian of this non-interacting system is written as

$$
\hat{H}_{\mathrm{ni}}=-\sum_{i}^{N} \frac{\hbar^{2}}{2 m_{e}} \nabla_{i}^{2}+\sum_{i}^{N} V_{\mathrm{eff}}\left(\mathbf{r}_{i}\right),
$$

where each term only operates on a single electron and is therefore separable. The total wave function for the system can thus be given as a determinant formed by the $N$ lowest solutions to the single-electron problem:

$$
\left(-\frac{\hbar^{2}}{2 m_{e}} \nabla^{2}+V_{\text {eff }}(\mathbf{r})\right) \phi_{i}(\mathbf{r})=\epsilon_{i} \phi_{i}(\mathbf{r}),
$$

The electron density of this non-interacting system, and as previously stated also that of the interacting system, is given by

$$
n(\mathbf{r})=\sum_{i}^{N}\left|\phi_{i}(\mathbf{r})\right|^{2} .
$$

Equations 3.16 and 3.17, together with the definition of $V_{\text {eff }}$ in Equation 3.14, make up the Kohn-Sham equations, which provides a path for finding the ground state electron density of a system. So far, no additional approximations have been introduced after the Born-Oppenheimer approximation, but before this method can be put to use in real life, two problems need to be addressed.

\section{The self-consistent field method}

The first problem lies in the fact that the potential $V_{\text {eff, }}$ defined in Equation 3.14 with a dependence on the electron density, is 


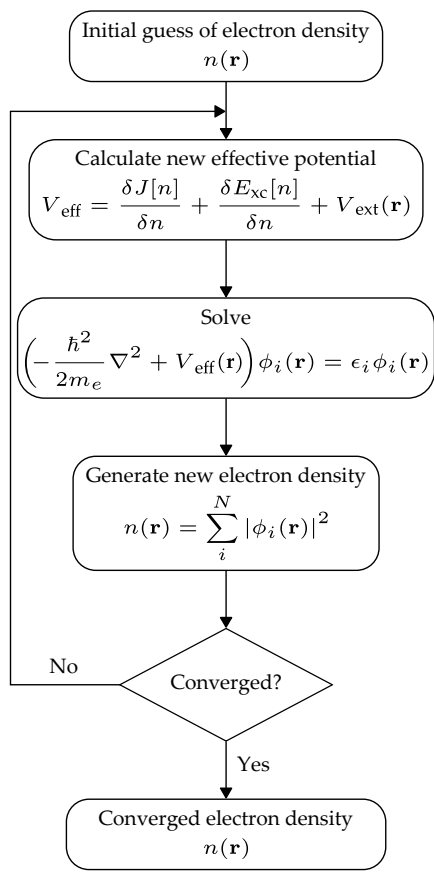

FIGURE 3.1: The self-consistent field method. necessary in order to solve Equation 3.16, the solutions of which are used in Equation 3.17 to obtain the electron density. The equations are non-linear and must therefore be solved in a selfconsistent manner. This means starting with some initial guess for the electron density, which is then inserted in Equation 3.14,

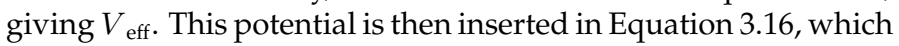
is solved to obtain the single-particle wave functions, $\phi_{i}$. These are then used to calculate a new electron density using Equation 3.17. The resulting electron density is then compared to that used to generate $V_{\text {eff. }}$. If they are the same, self-consistency has been achieved and the system has converged. If not, the calculated electron density is used to generate a new effective potential and the process is repeated. This scheme is known as the self-consistent field method and is summarized in Figure 3.1. In practice, the convergence criterion is weaker than that the initial and resulting electron densities should be equal, requiring only that they differ by less than some predefined value.

\section{Exchange-correlation functionals}

A more serious problem comes from the fact that the true form of the exchange correlation functional $E_{\mathrm{xc}}$, defined in Equation 3.12 is not known. Unlike the post-Hartree-Fock methods, where it is possible, at least in theory, to get as close as one wishes to the exact solution for a system by performing full configuration interaction calculations for an increasing basis set size, no such path exists for DFT. Rough guidelines for increasing the accuracy of the exchange-correlation functional have been suggested, however, such Jacob's ladder proposed by Perdew, ${ }^{26}$ shown in Figure 3.2, which contains a hierarchy of exchange-correlation functionals based on the variables on which they depend. Taking a step up on the ladder should generally, but is not guaranteed to, increase the quality of the calculated result while simultaneously increasing the required computational resources. In the following section, the main rungs of the ladder are examined.

In addition to which variables should be included, there are also different schools of thought as to how the functional should be constructed. ${ }^{27}$ It is inevitable that there will be some parameters in the functional, determining how the variables are used, and these must be chosen in some way. A number of properties are known for the exchange-correlation energy, such as the fact that it should be self-interaction free, meaning that the exchange and correlation parts should cancel for one-electron systems, and that a constant electron density should give the same result as for a uniform electron gas, the behaviour of which is known. Some functionals are based on these known facts, with parameters chosen to replicate limiting behavior. This does not, however, give any guarantee that the obtained results will be better for real molecular systems. The second school of thought is instead more 
interested in accurate results, and is willing to sacrifice the physicality of the description to attain them. In these functionals the parameters are instead fitted to recreate molecular properties for a set of reference molecules, obtained either through experiments or from high-quality calculations using other methods. This has the advantage of working well for molecules similar to those in the reference set, but with an unknown quality for those that are not.

\section{LOCAL DENSITY APPROXIMATION}

The first exchange-correlation functional, known as the local density approximation (LDA), was proposed by Kohn and Sham ${ }^{25}$ and is based on the idea that if the electron density varies slowly within a region, the exchange-correlation energy of that region can be approximated with that of a uniform electron gas of the same density. This assumption gives the following form for the exchange-correlation functional:

$$
E_{\mathrm{xc}}^{\mathrm{LDA}}[n(\mathbf{r})]=\int n(\mathbf{r}) \epsilon_{\mathrm{xc}}[n(\mathbf{r})] d \mathbf{r},
$$

where $\epsilon_{\mathrm{xc}}[n(\mathbf{r})]$ is the exchange-correlation energy per electron for a uniform electron gas of density $n(\mathbf{r})$. This can be split into a linear combination of the exchange energy, $\epsilon_{\mathrm{x}}$, and the correlation energy, $\epsilon_{\mathrm{c}}$. An analytical expression is known for the exchange energy, which has the form

$$
\epsilon_{\mathrm{x}}^{\mathrm{LDA}}=-\frac{3}{4}\left(\frac{3}{\pi}\right)^{\frac{1}{3}} n^{\frac{1}{3}},
$$

but the same is not true for the correlation energy, for which only the high and low density limits are known. Accurate correlation energy functions have been created, however, such as $\mathrm{VWN}^{28}$ and PW92 ${ }^{29}$, which are based on Quantum Monte Carlo calculations for a number of intermediate densities, interpolated by analytical functions. Due to the approximation that LDA is built upon, it works better for systems where the electron density varies slowly, such as for the valence electrons of solids. For molecules, where the electron density can change rapidly within small volumes, LDA is not a suitable choice.

\section{GENERALIZED GRADIENT APPROXIMATION}

Any real system will have a varying electron density and the standard way to expand the exchange-correlation functional to take this into account is to have the functional be dependent not just on the electron density, but also on its gradient, $\nabla n$. This is known as the generalized gradient approximation (GGA) and means that while the exchange-correlation contribution from a

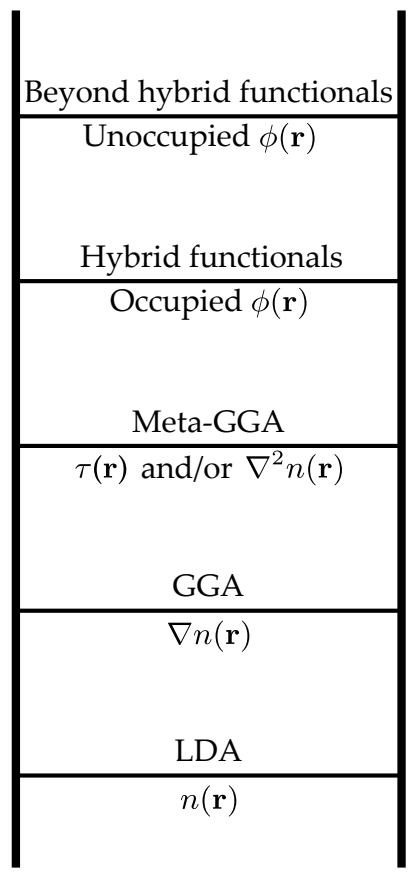

FIGURE 3.2: Jacob's ladder of exchange-correlation functionals. Accuracy and computational cost generally increase with each successive rung on the ladder. 
small volume element is still local and does not depend on the outside electron density, it does take into account the changes in density surrounding it through the derivatives. An examples of such a functional is the Becke $88^{30}$ exchange functional, which adds the gradient dependence as a correction to the LDA exchange energy as

$$
\begin{gathered}
\epsilon_{x}^{\mathrm{B} 88}=\epsilon_{x}^{\mathrm{LDA}}+\Delta \epsilon_{x}^{\mathrm{B} 88}, \text { where } \\
\Delta \epsilon_{x}^{\mathrm{B} 88}=-\beta n^{\frac{1}{3}} \frac{x^{2}}{1+6 \beta \sinh ^{-1} x} \text { and } x=\frac{|\nabla n|}{n^{\frac{4}{3}}} .
\end{gathered}
$$

This form of the functional contains a single parameter, $\beta$, which was obtained by fitting to calculated exchange energies for noble gases. On the correlation side, one popular GGA functional was developed by Lee, Yang and Parr (LYP), ${ }^{31}$ the form of which is rather long and will not be repeated here. This functional has four fitting parameters, determined from data for the helium atom. Creating the exchange-correlation functional is a simple matter of adding one exchange functional to one correlation functional, such as the common combination of the Becke88 exchange functional and LYP correlation functional to form the BLYP exchange-correlation functional.

GGA functionals generally give much better results than LDA for molecules, ${ }^{32}$ with improved accuracy for, among other things, total energies and energy barriers as well as binding energies, which LDA tends to overestimate. While the GGA functionals give better results than LDA, there are also a lot more of them, with increasingly complicated formulations for the exchange and correlation expressions and varying numbers of fitted parameters. Different functionals have different strengths and weaknesses depending on what they were parameterized for.

META-GENERALIZED GRADIENT APPROXIMATION

The natural extension of the GGA functionals is to also use the second order derivative of the electron density, $\nabla^{2} n$. It is also possible to use the kinetic energy density, $\tau$, defined as

$$
\tau(\mathbf{r})=\frac{1}{2} \sum_{i}\left|\nabla \phi_{i}(\mathbf{r})\right|^{2},
$$

which can be shown to contain the same information. Examples of such functionals include the $\mathrm{B}^{3} 5^{33}$ correlation functional and the full exchange-correlation functional M06-L. ${ }^{34}$

\section{HYBRID FUNCTIONALS}

The next rung of the ladder introduces non-local functionals, for which the exchange-correlation energy contribution from a point in space depends not just on the electron density in that specific 
point, but also those around it. This is done by including exact Hartree-Fock exchange energy, calculated based on the KohnSham orbitals, $\phi(\mathbf{r})$. This type of functional, known as hybrid functionals, were introduced by Becke, ${ }^{35}$ who used half of the Hartree-Fock energy and half of the LDA energy. Many different hybrid functionals have appeared since then, mixing and matching exact exchange with other exchange functionals at various ratios. The most successful of these, at least in terms of usage, is without a doubt B3LYP, ${ }^{36,37}$ which is extensively used in this thesis. It has the form

$$
\begin{aligned}
E_{\mathrm{xc}}^{\mathrm{B} 3 \mathrm{LYP}}= & E_{\mathrm{x}}^{\mathrm{LDA}}+a\left(E_{\mathrm{x}}^{\mathrm{HF}}-E_{\mathrm{x}}^{\mathrm{LDA}}\right)+b\left(E_{\mathrm{x}}^{\mathrm{B} 88}-E_{\mathrm{x}}^{\mathrm{LDA}}\right) \\
& +E_{\mathrm{c}}^{\mathrm{LDA}}+c\left(E_{\mathrm{c}}^{\mathrm{LYP}}-E_{\mathrm{c}}^{\mathrm{LDA}}\right),
\end{aligned}
$$

where the VWN functional is used for the LDA correlation and the three parameters, $a=0.20, b=0.72$ and $c=0.81$, were fitted to replicate atomic properties.

While there is no problem in finding functionals that outdo B3LYP for specific calculations, few are its equal when it comes to general performance. ${ }^{32}$ One of the things it does not do so well, which has become an issue in the work found in this thesis, is to describe electronic transitions and response properties of extended conjugated systems. ${ }^{38}$ This is due to an inaccurate description of long-range interactions, which is not an issue for smaller system, but can become one as the system grows. One way of dealing with this is to use a long-range corrected functional, such as CAM-B3LYP, ${ }^{39}$ which is also heavily used in this work. CAM-B3LYP uses an error function to smoothly increase the amount of Hartree-Fock exchange used in the functional for longer electron separation distances, creating a short-range behavior similar to B3LYP but with an altered long-range behavior that alleviates some of the problems found for larger systems.

\section{BEYOND HYBRID FUNCTIONALS}

One of the major failings of most DFT functionals is that they give poor descriptions of dispersive forces. ${ }^{27}$ Noble gases should be slightly attractive, but most functionals cause repulsive forces between the atoms, with the functionals that do generate an attraction underestimating it. The most common way to account for this is by adding an ad hoc dispersion correction term to the exchange-correlation energy, such as in the D $97^{40}$ functional, consisting of a sum of energy contributions for each atomic pair in the system. Each contribution depends on parameters fitted for the two participating atom types and is proportional to $R_{i j}^{-6}$, where $R_{i j}$ is the interatomic distance.

The fifth rung on the ladder suggests a more general approach that is expected to help in the description of dispersion forces. The idea is to not only use the occupied Kohn-Sham orbitals, 
as when calculating the Hartree-Fock exchange energy on rung four, but also the unoccupied ones. Work in this area is still in the early stages, however, and no commonly available functionals exist that take advantage of it. 


\section{RESPONSE THEORY}

A large number of properties can be measured for a system by seeing how it reacts to a small perturbing field, i.e. the response of the system. This is the main idea behind the field of response theory, in which the time-dependent behaviour of a system is studied as a function of an oscillating electromagnetic field. A significant discovery was made in this area in 1985 by Olsen and Jørgensen, ${ }^{41}$ who showed that excited state properties could be found within the response equations for ground state properties. While this is not strictly true in the case of time-dependent $\mathrm{DFT},{ }^{42}$ response theory does give access to approximate excitation energies and other properties that would otherwise be off limits due to the ground state nature of the theory.

This chapter contains a rough derivation of the response equations and the ideas behind them. While the derivation focuses exclusively on interactions with electric fields and stops at linear absorption, the response theory framework offers access to a large number of spectroscopies, both from interactions with electric as well as magnetic fields. The general principle is the same for all of them, though the expressions used to describe them become increasingly convoluted with higher orders. See the reviews of Norman ${ }^{43}$ and Helgaker et al. ${ }^{44}$ for a thorough survey of the history and current state of the field.

To begin with, the time-dependent Schrödinger equation is given by:

$$
i \hbar \frac{\partial}{\partial t}|\Psi\rangle=\hat{H}|\Psi\rangle \text {. }
$$

It is possible to split the Hamiltonian $\hat{H}$ into a time-independent and time-dependent part:

$$
\hat{H}=\hat{H}_{0}+\hat{V}(t) .
$$

Assuming that the time-dependent part, $\hat{V}(t)$, is small, it can be seen as a perturbation of the time-independent Hamiltonian, $\hat{H}$, meaning that the solutions of the time-dependent Scrödinger equation can be expressed in terms of the eigenstates of the unperturbed system:

$$
\hat{H}_{0}|n\rangle=E_{n}|n\rangle .
$$

In this case, the state of the system at time $t$ can expressed as

$$
|\Psi\rangle=\sum_{n} d_{n}(t) e^{-i E_{n} t / \hbar}|n\rangle,
$$

where the coefficients $d_{n}(t)$ isolate the time-dependent contribution from the perturbation and the requirement that $d_{n}(-\infty)=$ $\delta_{n 0}$ causes the system to start in the ground state. Applying the 
time-dependent Schrödinger equation to this wave function results in

$$
i \hbar \sum_{n} \frac{\partial}{\partial t}\left(d_{n}(t)\right) e^{-i E_{n} t / \hbar}|n\rangle=\sum_{n} \hat{V}(t) d_{n}(t) e^{-i E_{n} t / \hbar}|n\rangle .
$$

If the perturbation is due to an electric field, $F(t)$, the following interaction is obtained in the dipole approximation:

$$
\hat{V}(t)=-\hat{\mu}_{\alpha} F_{\alpha}(t) .
$$

Here, $\hat{\mu}_{\alpha}$ is the dipole moment operator and Einstein notation has been used to indicate summation over the three Cartesian axes. Inserting this into Equation 4.5 while multiplying both sides from the left with $\langle m| e^{i E_{m} t / \hbar}$ produces

$$
i \hbar \frac{\partial}{\partial t} d_{m}(t)=\sum_{n} F_{\alpha}(t) d_{n}(t) e^{i\left(E_{m}-E_{n}\right) t / \hbar}\left\langle m\left|\hat{\mu}_{\alpha}\right| n\right\rangle
$$

The coefficients $d_{n}(t)$ depend on the strength of the electric field, $F_{\alpha}(t)$, in some way and can be expanded into a power series as

$$
d_{m}(t)=d_{m}^{(0)}(t)+d_{m}^{(1)}(t)+d_{m}^{(2)}(t)+\cdots,
$$

where $d_{m}^{(k)}(t)$ depends on $\left(F_{\alpha}(t)\right)^{k}$. There is an extra $F_{\alpha}(t)$ on the right hand side of Equation 4.7, multiplied into $d_{n}(t)$, which allows a relationship to be found between $d_{m}^{(N)}(t)$ and $d_{m}^{(N+1)}(t)$ :

$$
\begin{aligned}
& d_{m}^{(N+1)}(t) \\
& =-\frac{1}{i \hbar} \int_{-\infty}^{t} \sum_{n} F_{\alpha}\left(t^{\prime}\right) d_{n}^{(N)}\left(t^{\prime}\right) e^{i\left(E_{m}-E_{n}\right) t^{\prime} / \hbar}\left\langle m\left|\hat{\mu}_{\alpha}\right| n\right\rangle d t^{\prime} .
\end{aligned}
$$

At this point the electric field is rewritten as a Fourier expansion, turning it into a sum of contributions from different frequencies. At the same time, a factor $e^{\epsilon t}$ is introduced, with an infinitesimal $\epsilon$. This ensures that the field has been slowly turned on at some point in the distant past, but that no memory of the event remains in the system. The electric field is thus described as

$$
F_{\alpha}(t)=\sum_{\omega} F_{\alpha}^{\omega} e^{-i \omega t} e^{\epsilon t}
$$

Inserting this into Equation 4.9 and introducing the transition angular frequency $\omega_{m n}=\left(E_{m}-E_{n}\right) / \hbar$ results in

$$
\begin{aligned}
& d_{m}^{(N+1)}(t) \\
& =-\frac{1}{i \hbar} \int_{-\infty}^{t} \sum_{\omega_{1}} \sum_{n} F_{\alpha}^{\omega_{1}}\left(t^{\prime}\right) d_{n}^{(N)}\left(t^{\prime}\right) e^{i \omega_{n m} t^{\prime}} e^{\epsilon t}\left\langle m\left|\hat{\mu}_{\alpha}\right| n\right\rangle d t^{\prime} .
\end{aligned}
$$


As the system starts in the ground state, the zero order coefficients must be $d_{m}^{(0)}(t)=\delta_{0 m}$, meaning the integration to find $d_{m}^{(1)}(t)$ can be carried out, resulting in

$$
d_{m}^{(1)}(t)=\frac{1}{\hbar} \sum_{\omega_{1}} \frac{F_{\alpha}^{\omega_{1}}\left\langle m\left|\hat{\mu}_{\alpha}\right| 0\right\rangle}{\omega_{m 0}-\omega_{1}-i \epsilon} e^{i\left(\omega_{m 0}-\omega_{1}\right) t} e^{\epsilon t} .
$$

At this point, Equation 4.11 can be used to produce increasingly complicated expressions for higher order coefficients. For this derivation, however, the first order coefficient is sufficient.

It is easy to see that the probability of finding the system in state $m$ at a given time $t$ must be proportional to $\left|d_{m}(t)\right|^{2}$. This makes it possible to study the likelihood that a photon will be absorbed based on the $d_{m}(t)$ coefficients, with one photon absorption connected to the $d_{m}^{(1)}(t)$ part, two-photon absorption to $d_{m}^{(2)}(t)$ and so on. Equation 4.12 can be written as

$$
d_{m}^{(1)}(t)=\frac{1}{\hbar} \sum_{\omega_{1}}\left\langle m\left|\hat{\mu}_{\alpha}\right| 0\right\rangle F_{\alpha}^{\omega_{1}} f\left(t, \omega_{m 0}-\omega_{1}\right),
$$

where $f\left(t, \omega_{m 0}-\omega_{1}\right)$ is a function that is sharply peaked when a frequency of the field, $\omega_{1}$, matches one of the transition frequencies of the system, $\omega_{m 0}$. The probability of absorption is thus high only when the absorbed energy is equal to one of the possible excitation energies and is proportional to the square of the corresponding transition dipole moment, $\left\langle m\left|\hat{\mu}_{\alpha}\right| 0\right\rangle$.

Continuing, the wave function is expanded in orders of the perturbing field in the same way as was done for the coefficients,

$$
|\Psi(t)\rangle=\left|\Psi^{(0)}(t)\right\rangle+\left|\Psi^{(1)}(t)\right\rangle+\left|\Psi^{(2)}(t)\right\rangle+\cdots,
$$

where each component can be written as

$$
\left|\Psi^{(N)}(t)\right\rangle=\sum_{n} d_{n}^{(N)}(t) e^{-i E_{n} t / \hbar}|n\rangle .
$$

This can then be used to write the expectation value of the dipole moment operator,

$$
\begin{aligned}
\left\langle\Psi(t)\left|\hat{\mu}_{\alpha}\right| \Psi(t)\right\rangle \\
=\left\langle\Psi^{(0)}(t)\left|\hat{\mu}_{\alpha}\right| \Psi^{(0)}(t)\right\rangle \\
\quad+\left\langle\Psi^{(1)}(t)\left|\hat{\mu}_{\alpha}\right| \Psi^{(0)}(t)\right\rangle+\left\langle\Psi^{(0)}(t)\left|\hat{\mu}_{\alpha}\right| \Psi^{(1)}(t)\right\rangle \\
\quad+\cdots \\
=\left\langle\mu_{\alpha}\right\rangle^{(0)}+\left\langle\mu_{\alpha}\right\rangle^{(1)}+\cdots,
\end{aligned}
$$

where the first term contains the unperturbed expectation value, the second contains the first order correction and so on. Using the expression for $d_{n}^{(1)}(t)$ that was found in Equation 4.12 and some simplification, the first two terms can be written as 


$$
\left\langle\mu_{\alpha}\right\rangle^{(0)}=\left\langle\Psi^{(0)}(t)\left|\hat{\mu}_{\alpha}\right| \Psi^{(0)}(t)\right\rangle=\left\langle 0\left|\hat{\mu}_{\alpha}\right| 0\right\rangle
$$

and

$$
\begin{aligned}
& \left\langle\mu_{\alpha}\right\rangle^{(1)} \\
& =\left\langle\Psi^{(1)}(t)\left|\hat{\mu}_{\alpha}\right| \Psi^{(0)}(t)\right\rangle+\left\langle\Psi^{(0)}(t)\left|\hat{\mu}_{\alpha}\right| \Psi^{(1)}(t)\right\rangle \\
& =\sum_{\omega_{1}} \frac{1}{\hbar} \sum_{n}\left[\frac{\left\langle 0\left|\hat{\mu}_{\alpha}\right| n\right\rangle\left\langle n\left|\hat{\mu}_{\beta}\right| 0\right\rangle}{\omega_{n 0}-\omega_{1}-i \epsilon}+\frac{\left\langle 0\left|\hat{\mu}_{\beta}\right| n\right\rangle\left\langle n\left|\hat{\mu}_{\alpha}\right| 0\right\rangle}{\omega_{n 0}+\omega_{1}+i \epsilon}\right] \\
& \quad \times F_{\beta}^{\omega_{1}} e^{-i \omega_{1} t} e^{\epsilon t} .
\end{aligned}
$$

Approaching this from another direction, the dipole moment of the system, $\mu(t)$, can be written in orders of the perturbing field

$$
\mu(t)=\mu_{0}+\alpha F(t)+\frac{1}{2} \beta F(t)^{2}+\frac{1}{6} \gamma F(t)^{3}+\cdots,
$$

where the polarizability, $\alpha$, first-order hyperpolarizability, $\beta$, and second-order hyperpolarizability, $\gamma$, have been identified, in addition to the permanent dipole moment, $\mu_{0}$. Using the Fourier decomposition of the field, the expression can be written as

$$
\begin{aligned}
\mu_{\alpha} & (t) \\
= & \mu_{\alpha}^{0} \\
& +\sum_{\omega_{1}} \alpha_{\alpha \beta}\left(-\omega_{1} ; \omega_{1}\right) F_{\beta}^{\omega_{1}} e^{-i \omega_{1} t} e^{\epsilon t} \\
& +\frac{1}{2} \sum_{\omega_{1}, \omega_{2}} \beta_{\alpha \beta \gamma}\left(-\omega_{\sigma} ; \omega_{1}, \omega_{2}\right) F_{\beta}^{\omega_{1}} F_{\gamma}^{\omega_{2}} e^{-i \omega_{\sigma} t} e^{2 \epsilon t} \\
& +\frac{1}{6} \sum_{\omega_{1}, \omega_{2}, \omega_{2}} \gamma_{\alpha \beta \gamma \delta}\left(-\omega_{\sigma} ; \omega_{1}, \omega_{2}, \omega_{3}\right) F_{\beta}^{\omega_{1}} F_{\gamma}^{\omega_{2}} F_{\delta}^{\omega_{3}} e^{-i \omega_{\sigma} t} e^{3 \epsilon t} \\
& +\cdots,
\end{aligned}
$$

where $\omega_{\sigma}$ is the sum of the other frequencies, i.e. $\omega_{1}+\omega_{2}$ for the $\beta$ terms and $\omega_{1}+\omega_{2}+\omega_{3}$ for the $\gamma$ terms. Comparing this expression with Equation $4.18, \alpha_{\alpha \beta}\left(-\omega_{1} ; \omega_{1}\right)$ can be identified as

$$
\begin{aligned}
& \alpha_{\alpha \beta}\left(-\omega_{1} ; \omega_{1}\right) \\
& \quad=\sum_{n \neq 0}\left[\frac{\left\langle 0\left|\hat{\mu}_{\alpha}\right| n\right\rangle\left\langle n\left|\hat{\mu}_{\beta}\right| 0\right\rangle}{\omega_{n 0}-\omega_{1}}+\frac{\left\langle 0\left|\hat{\mu}_{\beta}\right| n\right\rangle\left\langle n\left|\hat{\mu}_{\alpha}\right| 0\right\rangle}{\omega_{n 0}+\omega_{1}}\right],
\end{aligned}
$$

where the infinitesimal $\epsilon$ has been neglected and $n=0$ has been left out of the sum as in that case the two terms within the brackets cancel. It is easy to see here that the expression diverges every time the frequency of the field matches one of the transition frequencies. This means that it is possible to determine every single excitation energy just by searching for the poles of the polarizability. This is exemplified in Figure 4.1, in which the polarizability of one of the LCOs has been calculated over a range of field 
wavelengths, with the first excitation wavelength visible as a divergence of the curve. Furthermore, the residue corresponding to a pole provides the transition dipole moment of the excitation, which it was previously shown that the probability of absorption was proportional to. This can be used to construct the oscillator strength,

$$
f_{n 0}=\frac{2 m_{e}}{3 \hbar e^{2}} E_{n 0} \sum_{\alpha=x, y, z}\left|\left\langle 0\left|\hat{\mu}_{\alpha}\right| n\right\rangle\right|^{2},
$$

where $E_{n 0}$ is the excitation energy from the ground state to excited state $n$, while $m_{e}$ and $e$ are the electron mass and electron charge, respectively. The oscillator strengths are proportional to the probability of an absorption occurring, so when they are combined with the excitation energies, it is possible to construct the linear absorption spectrum of the system.

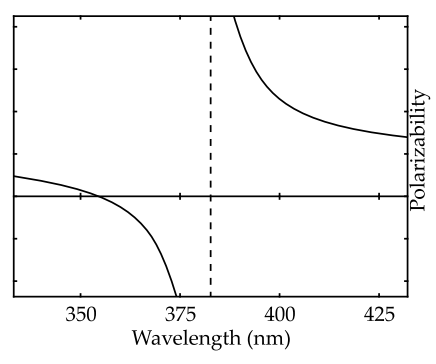

FIGURE 4.1: The polarizability of $\mathrm{p}$-HTAA, one of the LCOs, calculated over a range of field wavelengths. The divergence indicated by the dashed line corresponds to the excitation wavelength of the first excited state. 



\section{MOLECULAR MECHANICS}

Even with all the approximations and optimizations of the last hundred years, first principles quantum chemical calculations are still very resource demanding, even for quite small systems. The upper limit for a geometry optimization is around a thousand atoms and a dynamics simulation is only possible for systems of a few hundred atoms, and even then only for very short time scales. This means that unless new approximations are introduced, many systems that are of interest will be far beyond the capabilities of today's computers. A small piece of protein from the human body can consist of several thousand atoms, and even very small systems can increase by hundreds of atoms when a solvent is added. A popular way of dealing with this problem when simulating the motion of atoms is to use molecular mechanics (MM).

In electronic structure theory, the Born-Oppenheimer approximation is utilized to separate the Schrödinger equation into electronic and nuclear parts that can be solved separately. The electrons are approximated as responding instantly to any changes in the nuclear coordinates, allowing the electronic distribution to be found around stationary nuclei, after which the problem is solved for the nuclei moving in the effective potential generated by the electrons. Molecular mechanics takes this one step further and combines nuclei and electrons into a single unit, unsurprisingly referred to as atoms. The potential energy of the system is then approximated as a simple analytical function of the atomic coordinates. This potential energy function is split into contributions from different kinds of interactions involving two or more atoms, such as bond stretching and angle bending. The specific form of the potential energy function as well as the parameters used in it are know as a force field. The included interactions and the functional form that they take can differ depending on the requirements of the force field, with some focused on describing specific groups of molecules well and some sacrificing accuracy for computational efficiency. A simple energy function can look like

$$
E=E_{\mathrm{s}}+E_{\theta}+E_{\omega}+E_{\mathrm{vdw}}+E_{\mathrm{el}},
$$

where each term is a sum, adding upp all the contribution from a certain kind of bonded or non-bonded interaction in the system.

The potential energy function of the system has as its variables the atomic coordinates, but it also depends parametrically on how the atoms are bonded and the types of the involved atoms. In molecular mechanics, atom type does not just mean which element the atom belongs to, but also its chemical environment. This concept of atom types contains one of the main ideas behind MM force fields, which is that molecular structures that exist in 
similar environments behave in a similar manner. As an example, the carbon-carbon bond found in ethane is very similar to the two found in propane. The bond lengths are similar and the changes in potential energy when the bonds are stretched or contracted are almost the same. The parameters used in the potential energy function describing these three bonds can therefore safely be approximated as being the same. This transferability is one of the main strengths of using force fields, as parameters developed for a certain structure in one system can be reused for similar structures in other systems. For example, the parameters needed to describe butane are all that is required to describe all linear and branched alkanes in a simple force field.

While the carbon-carbon bonds in ethane and propane are similar enough for the parameters to be transferable, the same cannot be said for the carbon-carbon triple bond in acetylene, where the bond length is much shorter and the bond strength is significantly higher. Using the same parameters for all these bonds would lead to a poor description, which indicates that a finer division of atom types is needed than to just have one for each element. How fine this division is depends on what is desired from the force field. Creating accurate parameters can be a difficult and time-consuming process, with fittings made either to experimental data or higher level quantum mechanical calculations. A larger number of atom types makes it possible to give a more nuanced description of the interactions in the system, but it also strips away the transferability advantages. With more atom types the number of parameters quickly increases, and with them the likelihood that some of them will have to be created when studying a new system.

\section{Force field terms}

The following section describes some of the most commonly occuring force field interactions, using the $\mathrm{CHARMM}^{45}$ and $\mathrm{MM}^{46}$ force fields as examples. The two main categories of interaction terms are bonded and non-bonded. Bonded interactions exist for atoms that are part of the same molecule, the structure of which is defined at the start of the simulation by the user. Though not discussed in this thesis, there are also reactive force fields, such as ReaxFF, ${ }^{47}$ where bonds can be formed and broken dynamically. Non-bonded terms appear between atoms in different molecules as well as between atoms in the same molecule, provided that they are separated by at least a certain number of bonds - usually three. For atom pairs connected by fewer bonds, the non-bonded interactions are incorporated into the bonded interactions. 
Stretching or contracting a bond from its equilibrium position causes the energy of the system to rise. This change in energy is approximated by the bond stretch terms, usually represented by a simple harmonic oscillator of the form

$$
E_{\mathrm{s}}=\frac{k_{\mathrm{s}}}{2}\left(l-l_{0}\right)^{2},
$$

where $k_{\mathrm{s}}$ is a force constant, defining the strength of the bond, $l$ is the distance between the two bonded atoms and $l_{0}$ is the equilibrium distance for the interaction. Both $k_{\mathrm{s}}$ and $l_{0}$ are constants specific to the combination of atom types involved in the interaction. It should be noted that while $l_{0}$ is the bond length that minimizes Equation 5.2, it is not necessarily the equilibrium distance found in a molecule. As the two bonded atoms are most likely also involved in several other interactions, the molecular equilibrium distance will be the one that minimizes the full potential.

Generally, contracting a bond produces a steeper rise in energy than stretching it. This is accounted for in some force fields, such as MM3, which introduce anharmonicity into the bond energy expression by including higher order terms based on the Morse potential. ${ }^{48}$ The MM3 bond stretching term has the form

$$
E_{\mathrm{s}}=\frac{k_{\mathrm{s}}}{2}\left(l-l_{0}\right)^{2}\left(1-\alpha\left(l-l_{0}\right)+\alpha \frac{7}{12}\left(l-l_{0}\right)^{2}\right),
$$

where $\alpha$ is derived as 2.55 from the Morse potential, but could be taken as an additional parameter. Figure 5.2 shows the change in potential energy when stretching and contracting a hydrogencarbon bond in methane, calculated using an ab initio method as well as Equations 5.2 and 5.3. While the difference is significant for large distortions, the variations around the equilibrium are relatively small at room temperature, meaning the approximations are quite valid.

\section{ANGLE BENDING}

Same as for bond stretching, distorting an angle formed between two atoms bound to a common third atom from its equilibrium causes the energy of the system to rise. This too is commonly approximated by a harmonic oscillator of the form

$$
E_{\theta}=\frac{k_{\theta}}{2}\left(\theta-\theta_{0}\right)^{2}
$$

where $k_{\theta}$ is a force constant, $\theta$ is the angle formed by the atoms and $\theta_{0}$ is the equilibrium angle. The real angle bending interaction is clearly not a harmonic oscillator, as exemplified by the potential energy curve in Figure 5.4, showing the change in energy

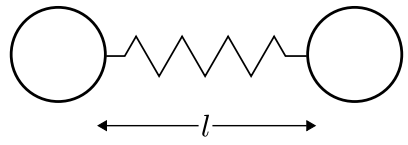

FIGURE 5.1: Bond stretching

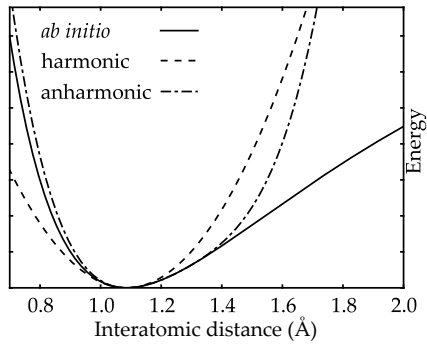

FIGURE 5.2: Potential energy curves for distortion of a methane $\mathrm{C}-\mathrm{H}$ bond. The reference $a b$ initio energy was calculated at the MP2/cc-pVTZ level of theory while the other curves were derived from the harmonic oscillator used in CHARMM and the anharmonic expression used in MM3. 


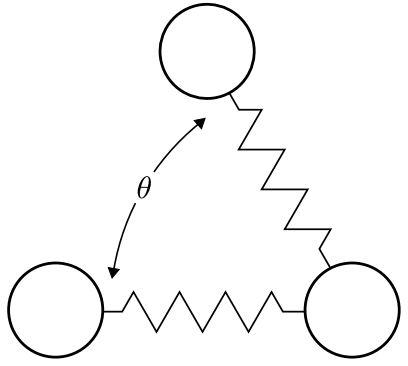

FIGURE 5.3: Angle bending

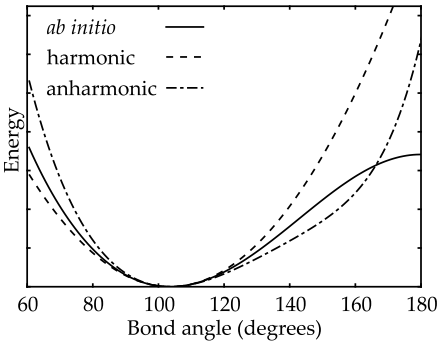

FIGURE 5.4: Potential energy curves for distortion of the water angle. The reference $a b$ initio energy was calculated at the MP2/cc-pVTZ level of theory while the other curves were derived from the harmonic oscillator used in CHARMM and the anharmonic expression used in MM3. when distorting the $\mathrm{H}-\mathrm{O}-\mathrm{H}$ angle found in water. Compressing the angle causes the two hydrogen atoms to come close together, leading to a steep curve near $\theta=0^{\circ}$, while increasing the angle leads to a cusp at $\theta=180^{\circ}$ due to symmetry. Again, some force fields account for this anharmonicity with additional terms, such as the MM3 angle bending term:

$$
\begin{aligned}
E_{\theta}=\frac{k_{\theta}}{2}\left(\theta-\theta_{0}\right)^{2}( & -\alpha\left(\theta-\theta_{0}\right)+\beta\left(\theta-\theta_{0}\right)^{2} \\
& \left.-\gamma\left(\theta-\theta_{0}\right)^{3}+\delta\left(\theta-\theta_{0}\right)^{4}\right),
\end{aligned}
$$

where the constants have been fitted to experimental data but are the same for all bending interactions. This gives a better agreement with the actual angle bending behaviour, as seen in Figure 5.4 , but again, for lower temperatures the harmonic oscillator is still a valid and computationally efficient approximation.

\section{TORSION}

When two atoms are bonded to atoms on opposite sides of a central bond, as for the opposing hydrogen atoms in ethane, then twisting these around the central bond can cause a change in the potential energy. The torsional energy is thus a function of the dihedral angle, $\omega$, formed between the first and fourth atom, while looking through the bond between the second and third atoms. For a visualization of this, see Figure 5.5. The torsion energy term is usually expressed in terms of a Fourier expansion of the form

$$
E_{\omega}=k_{\omega}(1+\cos (n \omega-\delta)),
$$

where $k_{\omega}$ is a force constant, $n$ is the periodicity and $\delta$ is a phase shift. Typically, several such terms are included for each set of four atoms in order to combine different multiplicities. The MM3 force field always includes terms of multiplicity 1, 2 and 3, with $\delta$ set to zero for each, while the CHARMM force field allows a more varied combination of terms.

Figure 5.6 shows the potential energy curve when twisting butane around the central carbon-carbon bond. The two identical lower peaks represent the conformations when the one hydrogen atom on each side is eclipsed by the carbon atom on the other side, while the main peak comes from the conformation when all atoms are eclipsed by an equivalent atom on the other side. It should be noted that the torsional energy of the force field does not come from a single interaction in this case, but nine. One for the two carbon atoms, four for the carbon atoms meeting the hydrogen atoms on the other side and four for the combinations of hydrogen atoms on opposite sides. It is easy to see that torsional interactions can quickly become very complicated when there are many atom types involved. This is further complicated 
by the fact that most force field also include non-bonded interactions between atoms separated by more than two bonds, meaning that the true rotational barrier will be a sum of all torsional and non-bonded interactions.

The conformation of a molecule is defined by its current dihedral angles, with two identifiable conformations for butane visible as minima in Figure 5.6: the global minimum at 180 degrees and two identical minima at \pm 70 degrees. For calculations where both the conformations themselves as well as the transitions between them are important, it is necessary to recreate the full potential energy curve and not just focus on the regions around the minima, as was done for bond stretching and angle bending terms.

\section{ELECTROSTATIC INTERACTION}

The non-bonded force field interactions are divided into electrostatic interactions, caused by static multipoles, and van der Waals interactions, containing everything else. For most force fields, only point charges are used in the electrostatic interactions, resulting in an energy given simply by the Coulomb interaction

$$
E_{\mathrm{el}}=\frac{q_{i} q_{j}}{4 \pi \epsilon_{0} r_{i j}},
$$

where $q_{i}$ and $q_{j}$ are the partial charges of the interacting atoms, $r_{i j}$ is the distance between them and $\epsilon_{0}$ is the vacuum permittivity. It is also possible to use higher order multipoles, an example of which can be found in the MM3 force field, where dipole moments are assigned to each bonded pair. This gives the advantage of a more flexible description, as the dipoles depend on the types of both involved atoms, but requires additional parametrization and has difficulty dealing with ionic systems.

\section{VAN DER WAALS INTERACTION}

The van der Waals interaction is typically represented by two terms. One due to exchange interaction, causing a strong repulsion at close distances, and one that is due to London dispersion, resulting in a weak attraction at longer distances. The most commonly used form is the Lennard-Jones potential: ${ }^{49}$

$$
E_{\mathrm{vdw}}=\epsilon\left[\left(\frac{r_{\mathrm{min}}}{r}\right)^{12}-2\left(\frac{r_{\mathrm{min}}}{r}\right)^{6}\right]
$$

where $\epsilon$ is the depth of the potential well, usually created as the mean of two parameters specific to the two interacting atom types, $r_{\min }$ is the position of the bottom of the well and $r$ is the interatomic distance. While it is possible to motivate the use of $r^{-6}$ for the attractive part, the use of $r^{-12}$ for the repulsive part is more pragmatic in origin, having that form simply because it

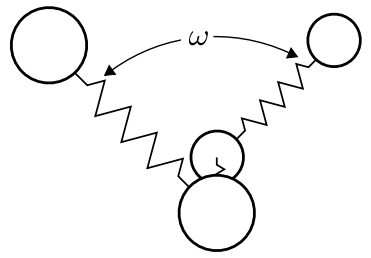

FIGURE 5.5: Torsion

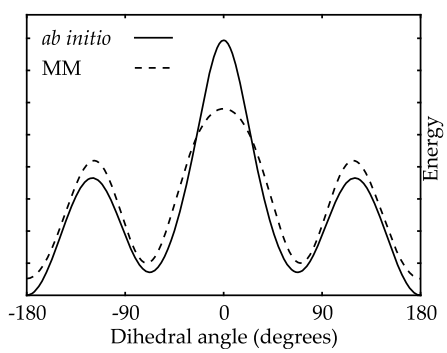

FIGURE 5.6: Potential energy curves for the torsional barrier found when twisting butane around the central bond. The reference $a b$ initio energy was calculated at the MP2/cc-pVTZ level of theory while the MM curve was obtained as a least squares fit of Equation 5.6 with multiplicities 1, 2, 3, 4 and 5 . 


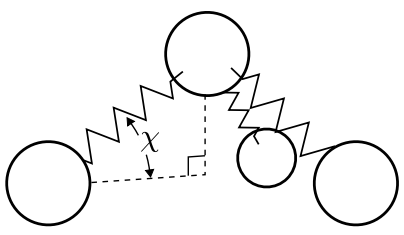

FIGURE 5.7: Out-of-plane bending is the square of $r^{-6}$, making its computation highly efficient. It can be argued ${ }^{27}$ that an exponential form is more realistic, such as the Buckingham potential ${ }^{50}$ used in MM3:

$$
E_{\mathrm{vdw}}=A e^{-B r}-\frac{C}{r^{-6}},
$$

where $A, B$ and $C$ are constants. Calculating this expression is more demanding than the Lennard-Jones potential, but produces a repulsive force that is closer to what is found using $a b$ initio methods. For very short interatomic distances, however, the Buckingham potential becomes strongly attractive, which can lead to highly unphysical behaviour for systems in unfavourable starting positions.

\section{ADDITIONAL TERMS}

Depending on what the force field is intended for, many additional terms can be added to the potential energy function. Crossterms are a common addition, accounting for more complex couplings between structural properties. Examples of these can be found in the MM3 force field, which contains stretch-bend, torsion-stretch and bend-bend interactions. As an example, the stretch-bend interaction accounts for the fact that compressing an angle formed by three bonded atoms will cause the repulsion between the two end atoms to increase, resulting in an elongation of their bonds to the middle atom. Another example of such structural corrections comes in the form of the out-of-plane term. If three or four atoms are bonded to a central atom with their equilibrium position in a plane, as for e.g. formaledehyde, the change in energy caused by a distortion of the central atom out of the plane is hard to model by just angle bending terms, as the individual angle distortions are small. The out-of-plane term instead depends on the angles formed between the plane and the bonds connecting the central and outer atoms. This is shown in Figure 5.7, where the $\chi$ is an example of an out-of-plane angle. 


\section{MOLECULAR DYNAMICS}

As described in previous chapters, the potential energy of a system can be obtained for a given set of nuclear positions either through QM methods or through more approximate means such as molecular mechanics. Given this knowledge, it is now possible to make the atoms move. Following the Born-Oppenheimer approximation, the behaviour of the nuclei should be described using quantum mechanics, but such calculations are too resource demanding for any larger systems. As such, this work deals only with classical molecular dynamics (MD), in which the nuclei move according to Newton's equations.

\section{Numerical integration}

\section{EULER INTERGRATION}

The first thing that is needed is the acceleration of each nucleus, obtained from the derivative of the potential energy function with respect to the nuclear coordinates. For the force field energy, a simple analytical function of $3 N$ coordinates, this is easily done, but for the quantum mechanics (QM) energy things are more complicated, possibly requiring numerical differentiation. Based on these derivatives, the acceleration for atom $j$ can easily be found using Newton's second law:

$$
\mathbf{a}_{j}=\frac{\mathbf{F}_{j}}{m_{j}}=-\frac{1}{m_{j}} \boldsymbol{\nabla}_{j} E
$$

where $m_{j}$ is the mass of atom $j$ and $\mathbf{F}_{j}$ are the forces acting on it.

If the atomic coordinates at a given time $t=t_{i}$ are $\mathbf{R}\left(t_{i}\right)$, the positions after a small time step, $\Delta t$, can be expressed as a Taylor expansion around $t=t_{i}$ :

$$
\begin{aligned}
& \mathbf{R}\left(t_{i}+\Delta t\right) \\
& =\mathbf{R}\left(t_{i}\right)+\frac{\partial \mathbf{R}}{\partial t} \Delta t+\frac{1}{2} \frac{\partial^{2} \mathbf{R}}{\partial t^{2}} \Delta t^{2}+\frac{1}{6} \frac{\partial^{3} \mathbf{R}}{\partial t^{3}} \Delta t^{3}+\cdots,
\end{aligned}
$$

where each partial derivative is evaluated at $t=t_{i}$. Given a fixed set of time steps, each of length $\Delta t$, a series of positions are obtained:

$$
\begin{aligned}
& \mathbf{R}_{i+1} \\
& =\mathbf{R}_{i}+\frac{\partial \mathbf{R}_{i}}{\partial t} \Delta t+\frac{1}{2} \frac{\partial^{2} \mathbf{R}_{i}}{\partial t^{2}} \Delta t^{2}+\frac{1}{6} \frac{\partial^{3} \mathbf{R}_{i}}{\partial t^{3}} \Delta t^{3}+\cdots \\
& =\mathbf{R}_{i}+\mathbf{v}_{i} \Delta t+\frac{1}{2} \mathbf{a}_{i} \Delta t^{2}+\frac{1}{6} \mathbf{j}_{i} \Delta t^{3}+\mathcal{O}\left(\Delta t^{4}\right),
\end{aligned}
$$

where $\mathbf{v}_{i}, \mathbf{a}_{i}$ and $\mathbf{j}_{i}$ are the velocities, acceleration and jerk of time step $i$, respectively. Truncating this to the first order gives

$$
\mathbf{R}_{i+1}=\mathbf{R}_{i}+\mathbf{v}_{i} \Delta t+\mathcal{O}\left(\Delta t^{2}\right),
$$




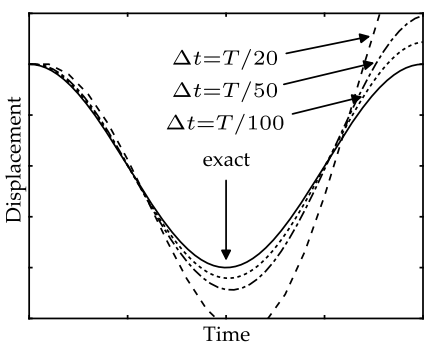

FIGURE 6.1: Simulation of a harmonic oscillator using Euler integration. The integration time step, $\Delta t$ is given as a fraction of the period of the oscillator, $T$.

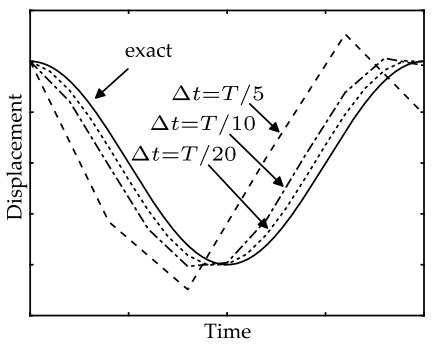

FIGURE 6.2: Simulation of a harmonic oscillator using Verlet integration. The integration time step, $\Delta t$ is given as a fraction of the period of the oscillator, $T$. which can be differentiated with respect to time to obtain the velocities for step $i+1$ :

$$
\begin{aligned}
\frac{\partial \mathbf{R}_{i+1}}{\partial t} & =\frac{\partial \mathbf{R}_{i}}{\partial t}+\frac{\partial \mathbf{v}_{i}}{\partial t} \Delta t+\mathcal{O}\left(\Delta t^{2}\right), \\
\mathbf{v}_{i+1} & =\mathbf{v}_{i}+\mathbf{a}_{i} \Delta t+\mathcal{O}\left(\Delta t^{2}\right) .
\end{aligned}
$$

These two equations, together with the expression for the acceleration found in Equation 6.1 make up the Euler method. For each step, the acceleration is obtained from Equation 6.1, then the velocities are calculated with Equation 6.5 and finally a new set of coordinates with Equation 6.4 before the process is repeated, step by step. This is an extremely simple method, with a local truncation error in order of $\Delta t^{2}$ for each step and a global error of first order for the trajectory as a whole. This can be seen from the fact that a trajectory of length $T$ requires $M=T / \Delta t$ steps. For each step, an error of order $\Delta t^{2}$ is added, leading to a total error of $T / \Delta t \mathcal{O}\left(\Delta t^{2}\right)=\mathcal{O}(\Delta t)$, i.e. first order. A simple example of integration using the Euler method is shown in Figure 6.1.

\section{VERLET INTERGRATION}

A better choice of integrator can be found in the Verlet algorithm. ${ }^{51}$ In this algorithm, the coordinates of the previous time step, $\mathbf{R}_{i-1}$, are required, which can be obtained by replacing $\Delta t$ with $-\Delta t$ in Equation 6.3:

$$
\mathbf{R}_{i-1}=\mathbf{R}_{i}-\mathbf{v}_{i} \Delta t+\frac{1}{2} \mathbf{a}_{i} \Delta t^{2}-\frac{1}{6} \mathbf{j} \Delta t^{3}+\mathcal{O}\left(\Delta t^{4}\right) .
$$

If Equations 6.3 and 6.6 are added together, the velocity and jerk terms cancel and the expression for $\mathbf{R}_{i+1}$ can be written as

$$
\mathbf{R}_{i+1}=\left(2 \mathbf{R}_{i}-\mathbf{R}_{i-1}\right)+\mathbf{a}_{i} \Delta t^{2}+\mathcal{O}\left(\Delta t^{4}\right) .
$$

Neglecting higher order terms and using the acceleration from Equation 6.1, this expression makes up the Verlet algorithm, with a local truncation error in the order of $\Delta t^{4}$. The global error, however, is of second order, ${ }^{27}$ due to the presence of two previous coordinates in the expression. Figure 6.2 shows the improvement over the Euler method.

\section{VELOCITY VERLET}

In any simulation that deals with temperature, the kinetic energy of the system becomes a factor, most commonly dealt with through the atomic velocities. As the Verlet algorithm neither calculates nor uses these velocities, measurements and alterations of the temperature become difficult. For this reason, the velocity Verlet algorithm ${ }^{52}$ was created. This uses the Taylor expansion of Equation 6.3 up to the second order for the coordinates:

$$
\mathbf{R}_{i+1}=\mathbf{R}_{i}+\mathbf{v}_{i} \Delta t+\frac{1}{2} \mathbf{a}_{i} \Delta t^{2}+\mathcal{O}\left(\Delta t^{3}\right) .
$$


Differentiating this expression with respect to time gives the expression for the velocity of the next step:

$$
\begin{aligned}
\frac{\partial \mathbf{R}_{i+1}}{\partial t} & =\frac{\partial \mathbf{R}_{i}}{\partial t}+\frac{\partial \mathbf{v}_{i}}{\partial t} \Delta t+\frac{1}{2} \frac{\partial \mathbf{a}_{i}}{\partial t} \Delta t^{2}+\mathcal{O}\left(\Delta t^{3}\right), \\
\mathbf{v}_{i+1} & =\mathbf{v}_{i}+\mathbf{a}_{i} \Delta t+\frac{1}{2} \mathbf{j}_{i} \Delta t^{2}+\mathcal{O}\left(\Delta t^{3}\right) .
\end{aligned}
$$

An expression for $\mathbf{j}_{i}$ can be found by differentiating once more and truncating after the second term:

$$
\begin{aligned}
\frac{\partial \mathbf{v}_{i+1}}{\partial t} & =\frac{\partial \mathbf{v}_{i}}{\partial t}+\frac{\partial \mathbf{a}_{i}}{\partial t} \Delta t+\mathcal{O}\left(\Delta t^{2}\right), \\
\mathbf{a}_{i+1} & =\mathbf{a}_{i}+\mathbf{j} \Delta t+\mathcal{O}\left(\Delta t^{2}\right) .
\end{aligned}
$$

This can be rearranged into

$$
\mathbf{j}_{i} \Delta t=\mathbf{a}_{i+1}-\mathbf{a}_{i}+\mathcal{O}\left(\Delta t^{2}\right),
$$

which can be inserted in Equation 6.9, resulting in

$$
\mathbf{v}_{i+1}=\mathbf{v}_{i}+\frac{1}{2}\left(\mathbf{a}_{i}+\mathbf{a}_{i+1}\right) \Delta t+\mathcal{O}\left(\Delta t^{3}\right) .
$$

Equations 6.1, 6.8 and 6.12 make up the velocity Verlet algorithm. First, Equation 6.8 is used to calculated the coordinates of the next step, which are used in Equation 6.1 to calculate the acceleration of that step. Finally, the velocities are calculated using Equation 6.12, completing the step.

As is evident from these equations, the local truncation error of the velocity Verlet algorithm is in the order of $\mathcal{O}\left(\Delta t^{3}\right)$, both for coordinates and velocities, but the global error is of second order, same as for the standard Verlet algorithm. This can be seen in Figure 6.3, where the total error of the velocity Verlet algorithm is comparable to that of the Verlet algorithm in Figure 6.2.

\section{Ensembles}

When simulating a system using molecular dynamics the ensemble of that system must be considered. That is, the states in which it is possible to find the system and how probable these are.

\section{THE MICROCANONICAL ENSEMBLE (NVE)}

Of the ensembles, the microcanonical is the simplest to simulate. Also known as the NVE ensemble, it represents a completely closed system, in which the number of particles, $N$, volume, $V$, and total energy, $E$, are kept constant. In simulation terms, this just means that the integration is left on its own from the starting position, with energy being converted back and forth between potential and kinetic form while the total sum remains constant. The main problem for NVE simulations is that it is hard

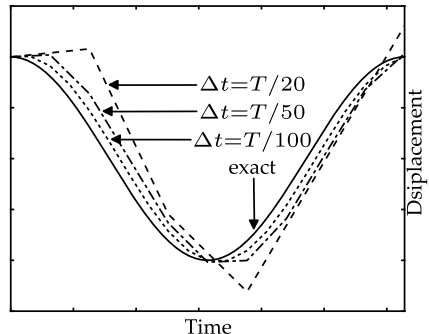

FIGURE 6.3: Simulation of a harmonic oscillator using velocity Verlet integration. The integration time step, $\Delta t$ is given as a fraction of the period of the oscillator, $T$. 
to maintain energy conservation when using numerical integration methods. As can be seen in the examples in the previous section, the accumulation of numerical errors causes a drift in energy proportional to the time step.

\section{THE CANONICAL ENSEMBLE (NVT)}

An ensemble that is of more practical use for realistic simulations is the canonical, or NVT, ensemble. In this case, the temperature is kept constant instead of the energy, emulating a system in thermal equilibrium with a heat bath. For the simulation, this means two things: First, the average temperature of the system should be constant. Note that this does not mean that the kinetic energy, the time average of which the temperature is proportional to, is constant at all times, just that it varies around the correct value. Second, how it varies should be determined by the fact that the velocities of the individual particles follow the MaxwellBoltzmann distribution. The task of maintaining these two criteria in an MD simulation is done by a thermostat.

The most basic thermostat is a simple rescaling of the system velocities by a factor

$$
\lambda=\sqrt{\frac{T_{0}}{T}},
$$

where $T$ is the current temperature and $T_{0}$ is the desired one. While this fulfils the criterion that the temperature should be constant, since the total kinetic energy is kept constant between steps, this also means that the second criterion is not fulfilled. An improved version of this method comes in the form of the Berendsen thermostat, ${ }^{53}$ which also includes a coupling parameter, $\tau$, determining the rate of heat transfer between the heat bath and the system. The rescaling factor then becomes

$$
\lambda=\sqrt{1+\frac{\Delta t}{\tau}\left(\frac{T_{0}}{T}-1\right)} .
$$

The coupling parameter needs to be chosen with care, as a $\tau$ that is equal to the time step, $\Delta t$, just reproduces the velocity rescaling thermostat and a too high $\tau$ makes the coupling too weak, with the limit $\tau \rightarrow \infty$ instead describing the microcanonical ensemble. So while the Berendsen thermostat does not describe a true canonical ensemble, there are coupling parameters between $\Delta t$ and infinity that give a suitably close approximation for larger systems. As the Berendsen thermostat is quite quick to converge to the correct temperature, it is often used for an initial equilibration, after which a true canonical thermostat is used.

Examples of such thermostats are found in the Andersen ${ }^{54}$ and Nosè-Hoover ${ }^{55,56,57}$ thermostats. The Andersen thermostat takes a stochastic approach, replacing the velocities of a selection 
of atoms at each step by new velocities taken from the MaxwellBoltzmann distribution, simulating random collisions. The frequency at which velocities are replaced becomes the coupling parameter of the thermostat, determining the speed at which it converges to the correct temperature. The Nosè-Hoover thermostat, on the other hand, introduces an artificial particle, with a mass and velocity, representing the heat bath. The extended system, containing both the real and artificial systems, is described by a microcanonical ensemble, but due to the coupling between the two, the real system becomes canonical.

ISOTHERMAL-ISOBARIC ENSEMBLE (NPT)

In the isothermal-isobaric, or NPT, ensemble, the pressure of the system is kept constant instead of the volume. This means that in addition to a thermostat regulating the temperature, a barostat is required to do the same for the pressure. For the three mentioned thermostats, there are corresponding barostats. In the case of the Berendsen barostat, the method is much the same as for the thermostat. Instead of velocities, however, it is the size of the periodic box containing the system and the coordinates within it that are scaled. If the pressure is too low, the box size is decreased and all atoms within it are brought closer to each other, and for a pressure that is too high the opposite is done. The Andersen and Nosè-Hoover barostats adopt a similar approach to the Nosè-Hoover thermostat, creating a coupling to an artificial system. This system acts as a piston, with artificial mass determining the strength of the coupling, trying to compress the real system.

\section{Geometry optimization}

A concept related to molecular dynamics is geometry optimization, which is the process of finding either the global minimum or a minimum close to a given geometry on the potential energy surface. With full knowledge of the potential energy function, this can be achieved analytically by first finding all stationary points, i.e. when the derivatives of the energy function with respect to the coordinates are all zero, and then finding the subset of those that have a positive definite Hessian matrix. For systems of any appreciable size, the number of coordinates makes such calculations difficult and the optimization are instead performed numerically. This is done using methods similar to those described for MD simulations, with properties calculated for the current geometry used to calculate the next set of coordinates. However, unlike in MD simulations, the path taken towards the minimum has no physical meaning and can differ significantly between methods. 
The simplest way of searching for a minimum is the steepest descent method, which finds the direction of the gradient vector for the energy function at the current point and takes a step in the opposite direction, with the step size depending on the length of the gradient vector. This method is guaranteed to approach a minimum, but will never reach it as the step size decreases the closer it gets. This is dealt with by monitoring the change in energy between steps and the forces acting on the system. If they are below certain criteria, a point close enough to the minimum has been found. The steepest descent method has a tendency to overshoot the minimum and oscillate back and forth over it for a long time, causing a slow convergence. Other options include the conjugate gradient method, which incorporates previous gradients into the calculation of the next step, and the Newton-Raphson method, in which the Hessian of the current point is used in addition to the gradient. Both of these methods improve on the convergence characteristics over the steepest descent method. However, as it has a quick convergence for the first few steps it may still be used at the beginning of an optimization before switching over to a more sophisticated method. 


\section{SOLVATION MODELS}

For systems that are too large for a QM description and calculations that require an accuracy that MM descriptions cannot provide, it may be a good idea to combine the two approaches. Often, only a small part of the system is of detailed interest, but if it is part of a larger molecule, or is surrounded by a solvent or other environment, this can affect the property that is being studied. In such cases it can be advantageous to split the system into several regions, described at different levels of theory. That way, the region of interest can be treated at a higher level while the surrounding environment is treated at a lower, more computationally effective level. This makes it possible to study much larger system without a significant loss in the quality of the results. This multiscale approach earned Karplus, Levitt and Warshel $^{58,59,60}$ the Nobel prize in chemistry in 2013.

There are two main approaches for dealing with solvation effects: atomistic models and continuum models. In atomistic models, the environment surrounding the QM region is described at the molecular mechanics level, with nuclei and electrons combined into classically described atoms. Because of this, models of this kind are often called quantum mechanics/molecular mechanics (QM/MM) models. In continuum models, the surrounding environment is instead represented by a homogeneous dielectric continuum. There are advantages and disadvantages to both approaches, making them suitable in different situations and for different systems.

With the QM/MM approach it is possible to describe complex inhomogeneous structures, such as proteins or other organic environments. It is also possible to treat systems where the QM region is part of a larger molecule that spans both regions. Furthermore, the QM/MM approach is especially suited for describing solvation effects where the discrete nature of the solvent molecules plays a role, causing an uneven distribution of charge around the solute. The drawback to this approach is that it is hard to find a single representative distribution of the solvent molecules around the solute for which to calculate the property of interest. To get around this, the typical approach is to create an average of the property, calculated for a number of structural snapshots taken from MD simulations, describing different distributions of the solvent around the solute. It is also possible to perform a single calculation on an averaged structure, ${ }^{61}$ though this removes some of the advantages of the model.

Continuum models take such averaging to the logical extreme, representing the region around the solute as a homogeneous medium, characterized by one or more dielectric constants, $\epsilon_{r}$. The advantage here is of course that no averaging is needed, as the dielectric medium already represents the average response of the 


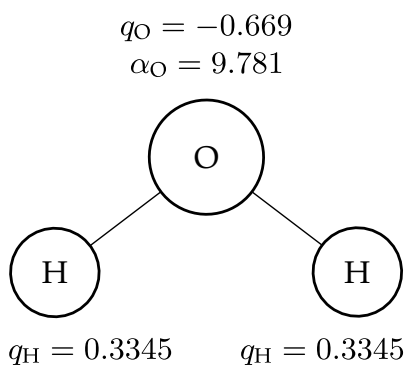

FIGURE 7.1: The polarizable water model used in this work. Charges are given in atomic units and polarizabilities in $\AA^{3}$.

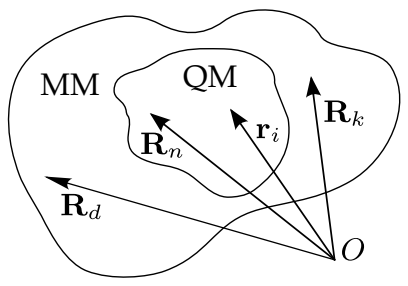

FIGURE 7.2: A QM/MM system, where the vectors $\mathbf{R}_{n}$ and $\mathbf{r}_{i}$ indicate the positions of the nuclei and electrons of the QM region, respectively, and $\mathbf{R}_{k}$ and $\mathbf{R}_{d}$ indicate the positions of the point charges and dipole moments of the MM region, respectively. environment. This, in addition to the low computational cost of the calculations and the fact that no force field description of the environment is needed, make such approaches an attractive alternative to $\mathrm{QM} / \mathrm{MM}$ when the discrete nature of the environment is not required.

In the following two sections, the general theory behind the two approaches is explained, with focus on the type of calculations done in this thesis.

\section{Quantum mechanics/molecular mechanics}

In this work, only systems where the individual molecules are fully inside one region are studied. Situations in which molecules span several regions are also possible to treat using QM/MM methods, see e.g. the review of Senn and Thiel, ${ }^{62}$ but require considerably more thought on how to deal with the connections between regions in a physically realistic way. The $\mathrm{QM} / \mathrm{MM}$ calculations performed in this work all deal with chromophores surrounded by a solvent, with the chromophore described at the QM level and the solvent molecules at the MM level. For water, the model of Ahlström et al., ${ }^{63}$ shown in Figure 7.1, has been used, which contains point charges and isotropic dipole polarizabilities. The following derivation assumes such a model, but expansions to higher order multipoles and anisotropic polarizibilities should pose no real trouble expect in the increasing number and complexity of the expressions. For a more in-depth look at such derivations, and for more technical details on the implementation of QM/MM energies and response functions, see e.g. the work of Kongsted and co-workers. ${ }^{64,65}$

Figure 7.2 shows a QM/MM system, consisting of a single QM region and a single MM region. For such a system, the Hamiltonian is given by

$$
\hat{H}=\hat{H}_{\mathrm{QM}}+\hat{H}_{\mathrm{QM} / \mathrm{MM}}+\hat{H}_{\mathrm{MM}},
$$

where $\hat{H}_{\mathrm{QM}}$ is the regular Hamiltonian that would have been used for the QM region in vacuum, $\hat{H}_{\mathrm{QM} / \mathrm{MM}}$ is the coupling between the QM and MM regions and $\hat{H}_{\mathrm{MM}}$ is the Hamiltonian of the MM part on its own, which is simply the potential energy function of the force field. The hard part of any QM/MM description lies in the coupling, $\hat{H}_{\mathrm{QM} / \mathrm{MM}}$, which in this case can be further decomposed as

$$
\hat{H}_{\mathrm{QM} / \mathrm{MM}}=\hat{H}_{\mathrm{vdw}}+\hat{H}_{\mathrm{el}}+\hat{H}_{\mathrm{pol}} .
$$

The first term, $\hat{H}_{\mathrm{vdw}}$, is computed entirely classically, with each atom in the QM region assigned a van der Waals parameter and the interaction energy between the atoms in the QM and MM regions calculated using the van der Waals term from the force 
field. For the positions of the atoms, those of the nuclei are used. As these, as well as the positions of the MM atoms, are kept fixed within the energy calculation, the contribution from the van der Waals term to the QM/MM Hamiltonian is constant.

The second term, $\hat{H}_{\mathrm{el}}$, is the electrostatic interaction between the nuclei and electrons found in the QM region and the point charges in the MM region. This is just a Coulomb interaction and can be calculated as

$$
\hat{H}_{\mathrm{el}}=\sum_{k=1}^{K} \sum_{n=1}^{N} \frac{q_{k} e Z_{n}}{4 \pi \epsilon_{0}\left|\mathbf{R}_{k}-\mathbf{R}_{n}\right|}-\sum_{k=1}^{K} \sum_{i=1}^{M} \frac{q_{k} e}{4 \pi \epsilon_{0}\left|\mathbf{R}_{k}-\mathbf{r}_{i}\right|},
$$

where vector definitions can be found in Figure 7.2. The index $k$ sums over point charges in the MM region, $n$ over the nuclei in the QM region and $i$ over the electrons in the QM region. The first term gives the interaction of the MM point charges with the nuclei in the QM region, which is a constant, and the second term does the same but for the electrons of the QM region.

The third term of the coupling Hamiltonian, $\hat{H}_{\text {pol }}$, is the interaction of the QM system with the induced dipole moments found in the MM region. This can be written as

$$
\begin{aligned}
& \hat{H}_{\mathrm{pol}} \\
& =-\frac{1}{2} \sum_{d=1}^{D} \boldsymbol{\mu}_{d}^{\text {ind }} \cdot\left(\sum_{i=1}^{M} \frac{-e\left(\mathbf{R}_{d}-\mathbf{r}_{i}\right)}{4 \pi \epsilon_{0}\left|\mathbf{R}_{d}-\mathbf{r}_{i}\right|^{3}}+\sum_{n=1}^{N} \frac{e Z_{n}\left(\mathbf{R}_{d}-\mathbf{R}_{n}\right)}{4 \pi \epsilon_{0}\left|\mathbf{R}_{d}-\mathbf{R}_{n}\right|^{3}}\right) \\
& \quad-\sum_{d=1}^{D}\left(\boldsymbol{\mu}_{d}^{\text {ind }}-\boldsymbol{\mu}_{d}^{\text {MMind }}\right) \cdot \sum_{k=1}^{K} \frac{q_{k}\left(\mathbf{R}_{d}-\mathbf{R}_{k}\right)}{4 \pi \epsilon_{0}\left|\mathbf{R}_{d}-\mathbf{R}_{k}\right|^{3}}
\end{aligned}
$$

which may require some explaining. The first term is the interaction of the induced dipoles, $\boldsymbol{\mu}_{d}^{\text {ind }}$, first with the electrons and then the nuclei of the QM region. The factor of one half that appears in front of the term comes from the energy required to create the dipoles. As the presence of the QM region changes the induced dipoles of the MM region, the interaction of those dipoles within the MM region is affected. This interaction is represented by the second term, which is the interaction of the induced dipoles with the point charges of the MM region. Here, $\boldsymbol{\mu}_{d}^{\mathrm{MMind}}$, is introduced, which is the dipole moment induced by the MM point charges. The interaction between these dipoles and the MM region would have existed even without the QM region, so it should be included in $\hat{H}_{\mathrm{MM}}$, not $\hat{H}_{\mathrm{QM} / \mathrm{MM}}$. For that reason, it is subtracted from $\boldsymbol{\mu}_{d}^{\text {ind }}$, leaving the part of the dipole induced by just the QM region.

In the linear approximation, the dipole moment induced by an electric field, $\mathbf{F}$, is given by

$$
\boldsymbol{\mu}=\alpha \mathbf{F},
$$


where $\alpha$ is the polarizability tensor, which for this isotropic case reduces to a constant, $\alpha_{d}$. With this relationship, expressions for $\boldsymbol{\mu}_{d}^{\text {ind }}$ and $\boldsymbol{\mu}_{d}^{\text {MMind }}$ can be found. The expression for the induced dipoles becomes

$$
\begin{aligned}
& \boldsymbol{\mu}_{d}^{\text {ind }} \\
& =\alpha_{d} \cdot\left[\sum_{i=1}^{M} \frac{-e\left(\mathbf{R}_{d}-\mathbf{r}_{i}\right)}{4 \pi \epsilon_{0}\left|\mathbf{R}_{d}-\mathbf{r}_{i}\right|^{3}}\right. \\
& \quad+\sum_{n=1}^{N} \frac{e Z_{n}\left(\mathbf{R}_{d}-\mathbf{R}_{n}\right)}{4 \pi \epsilon_{0}\left|\mathbf{R}_{d}-\mathbf{R}_{n}\right|^{3}}+\sum_{k=1}^{K} \frac{q_{k}\left(\mathbf{R}_{d}-\mathbf{R}_{k}\right)}{4 \pi \epsilon_{0}\left|\mathbf{R}_{d}-\mathbf{R}_{k}\right|^{3}} \\
& \left.+\sum_{d^{\prime} \neq d}^{D}\left(\frac{3\left(\mathbf{R}_{d}-\mathbf{R}_{d^{\prime}}\right)\left(\mathbf{R}_{d}-\mathbf{R}_{d^{\prime}}\right)^{\mathrm{T}}}{4 \pi \epsilon_{0}\left|\mathbf{R}_{d}-\mathbf{R}_{d^{\prime}}\right|^{5}} \boldsymbol{\mu}_{d^{\prime}}^{\text {ind }}-\frac{\boldsymbol{\mu}_{d^{\prime}}^{\text {ind }}}{4 \pi \epsilon_{0}\left|\mathbf{R}_{d}-\mathbf{R}_{d^{\prime}}\right|^{3}}\right)\right]
\end{aligned}
$$

where the final sum gives the electric field of all the other dipoles. This dependency of the induced dipoles on themselves means that the solution is typically found in a self-consistent manner. Similarly, it can be can be shown that

$$
\begin{aligned}
& \boldsymbol{\mu}_{d}^{\text {ind }}-\boldsymbol{\mu}_{d}^{\text {MMind }} \\
& =\alpha_{d}\left(\sum_{i=1}^{M} \frac{-e\left(\mathbf{R}_{d}-\mathbf{r}_{i}\right)}{4 \pi \epsilon_{0}\left|\mathbf{R}_{d}-\mathbf{r}_{i}\right|^{3}}+\sum_{n=1}^{N} \frac{e Z_{n}\left(\mathbf{R}_{d}-\mathbf{R}_{n}\right)}{4 \pi \epsilon_{0}\left|\mathbf{R}_{d}-\mathbf{R}_{n}\right|^{3}}\right) .
\end{aligned}
$$

Equations 7.6 and 7.7 can be inserted into Equation 7.4, which can itself be inserted into Equation 7.2, providing the full coupling Hamiltonian.

\section{Continuum models}

Any continuum model calculation starts with the creation of a cavity around the solute. This cavity is supposed to represent the border between the solute on the inside and the solvent on the outside and can be created in a number of ways. The most basic method for generating a cavity is to simply place a sphere or an ellipsoid around the solute, though this is likely to create large empty spaces within the cavity for unevenly shaped solutes. A slightly better cavity can be found by placing a sphere around each nucleus, with the radius taken as the van der Waals radius of the atom, scaled slightly larger by a constant factor. The union of these spheres generates the van der Waals surface, which is computationally efficient but can contain unnatural crevices where a solvent molecule would not fit. This can be addressed by instead using the solvent accessible surface (SAS), ${ }^{66,67}$ which can be obtained by rolling a spherical probe over the solute and tracing its centre. As the radius of the probe depends on the size of the solvent, the shape of the cavity depends both on the solute and the solvent surrounding it. This can be further improved by tracing 
the inward facing part of the probe, creating the solvent excluded surface (SES). ${ }^{68}$ Figure 7.3 shows examples of these cavities and how they are formed. The next step is to approximate the cavity by tesselating the spheres, creating a three-dimensional mesh of flat polygons. Depending on the desired quality of the calculation, the surface area of these elements, called tesserae, can be varied, creating a finer or coarser mesh. A finer mesh means more accurate calculations, but also higher computational demands.

One of the most commonly used continuum models is the polarizable continuum model (PCM) ${ }^{69}$ and the following derivation follows the general theory of that method. The charge density, $\rho_{s}$, at a specific point on one side of a surface can be derived from electromagnetic relations as $\rho_{s} / \epsilon_{0}=\mathbf{F} \cdot \hat{\mathbf{n}}$, where $\mathbf{F}$ is the electric field just outside the surface, $\hat{\mathbf{n}}$ is the normal of the surface at the given point and $\epsilon_{0}$ is the vacuum permittivity. The total surface charge density at the point then becomes

$$
\frac{\rho_{s}}{\epsilon_{0}}=\mathbf{F}_{\text {in }} \cdot(-\hat{\mathbf{n}})+\mathbf{F}_{\text {out }} \cdot \hat{\mathbf{n}},
$$

where $\mathbf{F}_{\text {in }}$ and $\mathbf{F}_{\text {out }}$ are the electric fields on the inside and outside of the surface, respectively. In the linear response limit, $\epsilon_{\text {in }} \epsilon_{0} \mathbf{F}_{\text {in }}=\epsilon_{\text {out }} \epsilon_{0} \mathbf{F}_{\text {out }}$, where $\epsilon_{\text {in }}$ and $\epsilon_{\text {out }}$ are the relative permittivities of the materials inside and outside the surface, respectively. There is a vacuum on the inside of the surface, which has a relative permittivity of 1 , and on the outside there is a solvent with a relative permittivity $\epsilon_{r}$, giving $\mathbf{F}_{\text {in }}=\epsilon_{r} \mathbf{F}_{\text {out }}$. Dropping the subscript for $\mathbf{F}_{\text {out }}$, Equation 7.8 can the be rewritten as

$$
\rho_{s}=-\epsilon_{0}\left(\epsilon_{r}-1\right) \mathbf{F} \cdot \hat{\mathbf{n}} .
$$

If each tessera is small enough then the surface charge can be considered constant on the whole surface, and the field created by each of them can be approximated as a point charge with charge

$$
q_{i}=S_{i} \rho_{i}=-S_{i} \epsilon_{0}\left(\epsilon_{r}-1\right) \mathbf{F}_{i} \cdot \hat{\mathbf{n}}_{i},
$$

where $S_{i}$ is the surface area of tessera $i, \rho_{i}$ is the surface charge at a representative point in the middle of the tessera and $\hat{\mathbf{n}}_{i}$ is the normal of the surface at that point. The electric field at point $i, \mathbf{F}_{i}$, is created both by the solute and the point charges representing all the tesserae. Inserting an expression for the electric field into Equation 7.10 results in

$$
\begin{aligned}
q_{i}= & -S_{i} \epsilon_{0}\left(\epsilon_{r}-1\right)\left(1+\eta_{i}\right) \\
& \times\left(\mathbf{F}_{\text {solute }}\left(\mathbf{R}_{i}\right)+\sum_{j \neq i} \frac{q_{j}}{4 \pi \epsilon_{0}\left|\mathbf{R}_{i}-\mathbf{R}_{j}\right|^{3}}\left(\mathbf{R}_{i}-\mathbf{R}_{j}\right)\right) \cdot \hat{\mathbf{n}}_{i}
\end{aligned}
$$

where the first term within the parenthesis comes from electric field caused by the solute and the second term results from the

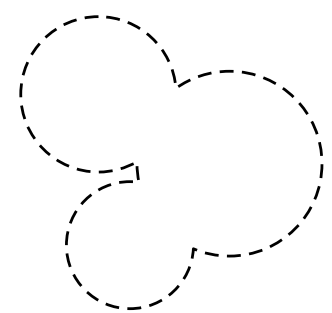

van der Waals surface

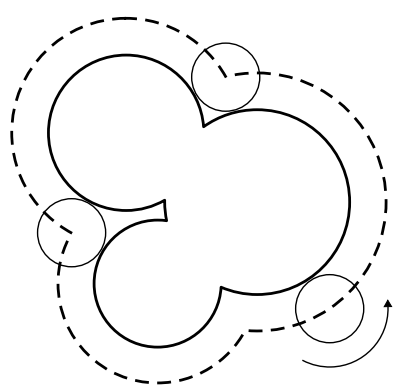

Solvent accessible surface

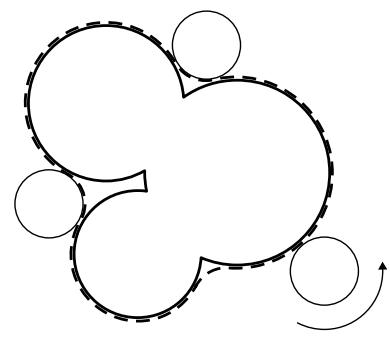

Solvent excluded surface

FIGURE 7.3: Examples of methods for generating cavities. 
electric field caused by the other tesserae. The factor $1+\eta_{i}$ has been added to compensate for the fact that the surface is not flat, but slightly concave or convex, increasing its area. Equation 7.11 creates a large linear equation system, where each charge, $q_{i}$ depends on every other charge. Depending on the size of the equation system, i.e. the number of tesserae, this can be solved either through matrix inversion or iterative methods, giving a final set of point charges which can be included when solving the solute system. As point charges representing the nuclei are already included, this can easily be implemented.

Another example of a continuum model and an alternative to PCM is the conductor-like screening model (COSMO). ${ }^{70}$ This model treats the dielectric medium as an ideal conductor, i.e. with a relative permittivity of infinity. This greatly reduces the computational cost of calculating the surface charges, which are later scaled by a factor that depends on the true permittivity of the solvent. This approximation works better for solvents with higher relative permittivities such as water, which are closer in behaviour to the approximated system. 


\section{CONFORMATIONAL AVERAGING}

Using the methodology described in the previous chapters it is possible to calculate the transition energies and relative strengths of absorption or emission for a molecule, which can then be used to construct the spectrum. The calculated properties depend parametrically on the geometry of the molecule, and the subject of this chapter is the question of what the geometry should be.

When a spectrum is measured experimentally, it is not done for a single molecule and it is not individual absorption or emission events that are measured. Instead, the measurement is performed on a sample containing vast amounts of molecules, irradiated by many photons over a period of time. The resulting spectrum thus becomes an average both over time as well as the individual molecules. In calculations, this is most easily taken into account for rigid molecules, where a single representative optimized structure can be found. There are still geometry variations for such molecules, but they are small distortions around the optimized structure, causing only slight spectral changes. This is typically taken into account by a simple broadening of the spectrum for the optimized structure. As the size of the geometry distortions increase with the temperature of the system, the amount of broadening applied to the spectrum should also depend on the temperature.

\section{Boltzmann averaging}

For semi-rigid molecules, where there are multiple well defined conformations in which the molecule can be found, the spectral differences between conformations may be significant. For this reason, a single broadened spectrum is most likely not representative of the molecule as a whole, meaning an average has to be used instead. As the probabilities of finding the molecule in specific conformations are most likely not equal, a weighted average has to be used. The most common method for generating these weights is to use the Boltzmann distribution, which gives the relative probability of a state based on its energy and its temperature. Given a known set of conformations, the probability of finding the molecule in conformation $n$ is

$$
P_{n}=\frac{e^{-\frac{E_{n}}{k_{\mathrm{B}} T}}}{\sum_{i} e^{-\frac{E_{i}}{k_{\mathrm{B}} T}}},
$$

where $E_{n}$ is the energy of conformation $n, k_{\mathrm{B}}$ is the Boltzmann constant and $T$ is the temperature.

While Boltzmann weights are easy to obtain, they only consider the local minima points on the potential energy surface and 


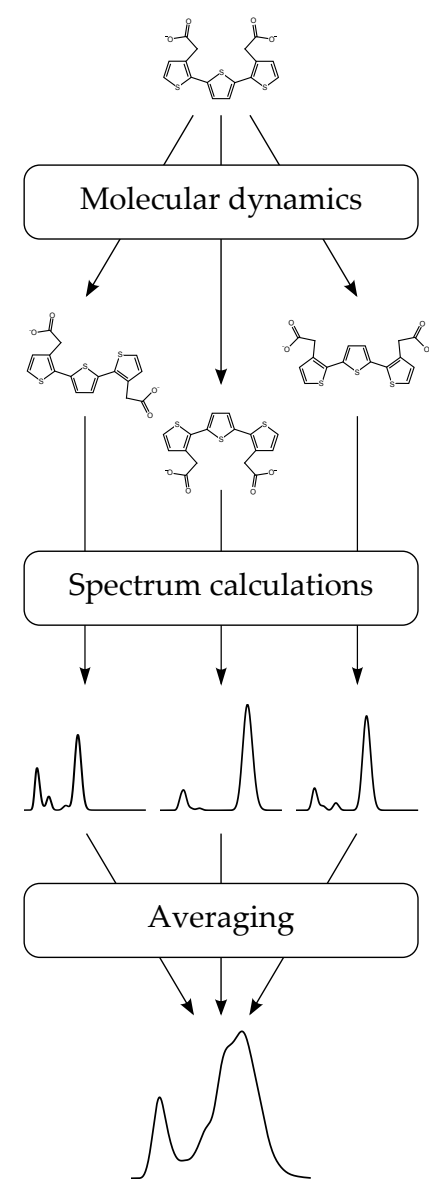

FIGURE 8.1: Scheme for conformational averaging using MD sampling. not their surroundings. For more flexible molecules, the curvature around the minima might have a significant impact on the conformational weights. This can be taken into account by examining statistics from a molecular dynamics simulation. Assuming an accurate enough description of the system in the simulation, the conformational weights can be obtained by counting the number of frames in the MD trajectory that correspond to each conformation and then dividing by the total number of frames. This method is used in Paper V to weight IR and Raman spectra.

\section{Molecular dynamics sampling}

For larger and more flexible molecules, for which there is a large number of less well defined conformations, the use of weighted spectra for optimized structures does not work very well. In that case, the use of MD simulations can be taken one step further, using not just the conformational statistics but also the full geometry of the samples.

Some criteria need to be fulfilled before the sampled structures from the MD trajectory can be put to use. First, the description of the system in the force field must be of high quality, ensuring that the potential energy surface that the molecule moves on is accurately reproduced. Second, the MD simulation must be long enough that this potential energy surface can be fully explored. Third, the number of samples taken from the trajectory must be large enough that it accurately represent the conformational distribution of the full simulation. Assuming all these criteria are fulfilled, a sampling of the MD trajectory will yield structures that are comparable to the distribution of conformations found in an experimental sample. Thus, by calculating a spectrum for each of the sampled structures and averaging these, a spectrum is obtained that represents the experimentally measured spectrum. This scheme is illustrated in Figure 8.1. What constitutes a long enough simulation time and a large enough sample size depends on the system that is under study. Both the size of the molecule, which determines the size of the potential energy surface that must be explored, as well as its flexibility, which determines how fast that exploration will occur, play a role. For the highly flexible systems studied in this work, sample sizes of a few hundred structures have been used, taken from simulations of at least several hundred picoseconds.

An added benefit to this method is that the influence of the environment on the spectrum can easily be taken into account. The environmental effects can be split into two parts, direct and indirect, depending on the manner in which they influence the calculated spectrum. Most of the time, the system of interest is not in the gas phase, meaning that it will be constantly interacting with the molecules that surround it. The changes that these interactions cause in the conformational distribution, and in turn 
the spectrum, are the indirect environmental effects. These are included in the calculation of the averaged spectrum by ensuring that the environment used in the MD simulations corresponds to that of the real system and that it is well described in the force field. As discussed more in depth in the chapter on solvation models, the presence of surrounding molecules alters the electronic distribution of the solute. The changes this causes in the spectrum are the direct effects, and as described in that chapter, there are a number of models for including them in the spectrum calculations. For the described method of structural sampling, the QM/MM model is especially suitable as it benefits from the fact that not just the structure of the molecule of interest is sampled, but also that the environment surrounding it.

In Papers II and III of this thesis, structural sampling is used to calculate fluorescence spectra. While this is perfectly possible, it does require some additional considerations. First, a description of the excited state from which the fluorescence occurs is needed in the force field. One of the main concepts behind molecular mechanics is that similar structures of atoms behave in roughly the same way, meaning parameters created for one structure can be reused for all others of the same type. Due to the changes in electronic structure in the excited state, possibly only localized to some parts of the molecule, there is no guarantee that any of the ground state parameters will still give an accurate representation of the system. This means that new parameters have to be created specifically for the molecule and excited state in question, a process that can be both difficult and tedious.

The second aspect that needs to be considered when calculating fluorescence spectra is that the sampling cannot necessarily be done in the same way as for absorption. The time the molecule spends in the excited state before emitting the photon again, known as the fluorescence lifetime, is typically between a few hundred and a few thousand picoseconds. Depending on the shape of the potential energy surface in the excited state, this is not guaranteed to be enough time to properly explore the conformational space. It may not even be enough to get out of the conformation in which it starts. In that case there is a correlation between the conformations in which absorption and emission occur, meaning that samples collected from a single excited state dynamic will not necessarily represent the correct conformational distribution.

In Papers II and III, this is dealt with in two different ways, the first of which is a robust method that simulates every step of the fluorescence process. This is done by first collecting atomic velocities in addition to positions when conducting the sampling of a ground state dynamic for an absorption spectrum. Each sampled point then acts as a starting point for a separate excited state dynamic, run for a length of time equal to the fluorescence lifetime. The structures found at the end of these dynamics are saved and 


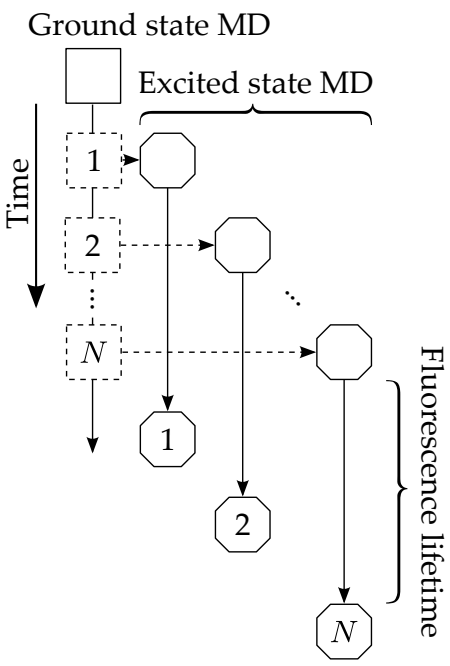

Ground state samples

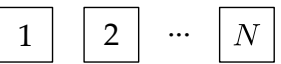

Excited state samples

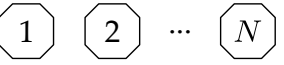

FIGURE 8.2: Scheme for generating structural snapshots for use in fluorescence calculations. used as samples for the fluorescence calculations. This way, an equal number of ground and excited state snapshots are obtained and the full fluorescence process is simulated, from excitation to relaxation in the excited state and finally deexcitation. Figure 8.2 illustrates how this scheme is used to obtain excited state structural snapshots.

For the LCO studied with this method, it was observed that the excited state simulations all ended up in one of three conformations and that this conformation was fully correlated to the structure in which the simulation started. It was also noted that relaxation occurred very quickly, with no memory of the starting position except the general conformation remaining at the end of the excited state dynamic. Using this information, a different approach was employed for the fluorescence calculation in the following paper. The structural samples were then taken from three excited state MD simulations, one for each conformation, in proportion to the ground state conformational distribution. This method, while significantly less general, reduces the number of required excited state simulations by a factor of one hundred. 


\section{PROTEIN INTERACTION}

The ultimate aim of the studies conducted on luminescent conjugated oligothiophenes in Papers II-IV has been to explain the significant changes in luminescence properties that occur when the probes bind to amyloid proteins. While Paper IV does make predictions about this cause based on simulations in solvent, there are no studies of the probes interacting with the protein in the published material. This does not mean that no such simulations have been performed, just that their outcomes were less than satisfactory. This chapter takes the opportunity to put to paper these, along with other equally inconclusive results found over the course of our investigations. Figure 9.1 shows the molecular structures of the LCOs used in this chapter.

\section{Planarization}

The current hypothesis ${ }^{3,71}$ for what occurs with the probes upon binding is that they become conformationally restricted in a protein binding pocket, causing a planarization of the conjugated backbone and a redshift of the absorption spectrum. This theory is corroborated by the results found in Paper IV, in which it is shown that not only is a planarization capable of producing the observed spectral shifts, the structural distortions required to achieve this are well within realistic limits.

\section{PROTEIN DOCKING}

To investigate whether such a docking site could be found, several simulations of LCOs together with amyloid proteins have been carried out. The question of what protein structure to use is a somewhat tricky one, as amyloid proteins are defined by their secondary, tertiary and quaternary structures and not their primary structure. The secondary structure is that of a folded $\beta$ sheet, shaped like a hairpin, a large number of which are stacked on top of each other in the tertiary structure, forming long fibrils. These fibrils are then twisted around each other, forming aggregate bundles known as amyloids. This is illustrated in Figure 9.2. In the performed calculations, a model of a protein associated with Alzheimer's disease is used. ${ }^{72}$ This model provides the primary, secondary and a beginning of the tertiary structure for the fibril, including five stacked $\beta$-sheets. While the tertiary structure of the protein is easy to extrapolate based on the twist and distance between the included $\beta$-sheets, there is no way to do the same for the quaternary structure. There have been studies giving rough fittings of known structures to fibril aggregates, ${ }^{73}$ but no models suitable for use in molecular mechanics simulations currently exist. The amount of work required to construct
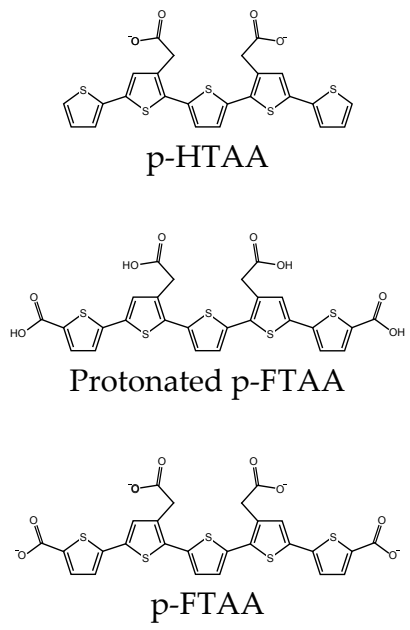

FIGURE 9.1: The LCOs used in this chapter.

Primary structure

-Leu-Val-Phe-Phe-Ala-Glu-

Secondary structure

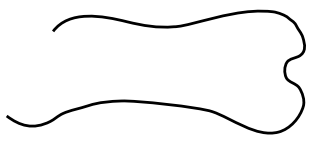

Tertiary structure

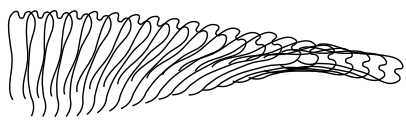

Quaternary structure

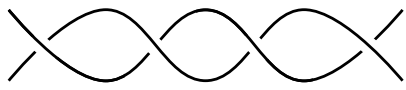

FIGURE 9.2: Structure levels of amyloid proteins. 


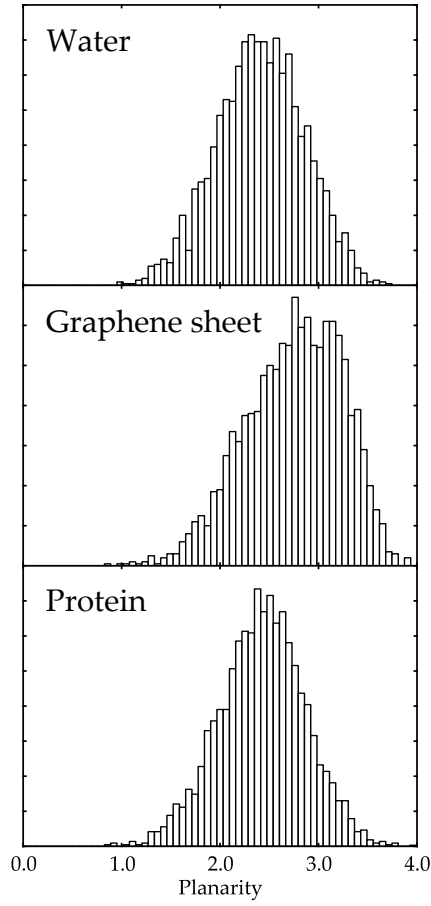

FIGURE 9.3: Distribution of planarity for 2500 structural samples, taken from MD simulations of p-FTAA in water solution, together with a graphene sheet and together with an amyloid protein.

(I) The simulations were run for a single p-HTAA molecule, docked to a protein fibril constructured from $50 \beta$-sheet strands, solvated in water and described by the CHARMM force field. such a model would in all likelihood fill another thesis, so all simulations have been conducted using the single fibril structure available. This carries with it a certain amount of uncertainty, as any negative results could simply be due to the fact that binding occurs between the fibrils of the quaternary structure, a situation which cannot be captured by the current model.

Using the Autodock ${ }^{74}$ program, which searches for sites on proteins to which a specified ligand can be bound, a number of possible docking sites for one of the LCOs, p-HTAA, were found on the fibril. Using these structures as starting points for MD simulations, ${ }^{\text {I }}$ it was found that the ligand left the binding site almost immediately, staying only a few picoseconds and showing no signs of conformational change. While the failure of the protein to retain the probes may be due to inadequacies in the force field description, a more likely explanation is that there are no docking sites on an individual fibril capable of holding the LCOs for any appreciable length of time. Instead, the many crevasses and spaces found in the fibril bundles seem like a more likely source of suitable binding pockets.

\section{SURFACE PLANARIZATION}

In Paper IV, a measure of planarity is defined for the LCOs as

$$
P=\sum_{i} \frac{|| \theta_{i}|-90|}{90}
$$

where $\theta_{i}$ is one of the dihedral angles formed between two of the thiophene rings and the sum is over all such angles. This measure gives a value between 0 and $N-1$, where $N$ is the number of thiophene rings in the $\mathrm{LCO}$, with increasing $P$ indicating a more planarized system. For a given LCO geometry, $P$ shows a strong correlation to the energy of the first electronically excited state, which is dominant in the absorption spectrum. Using this correlation, it is possible to make predictions regarding the spectral properties of an LCO based only on the distribution of $P$ extracted from an MD simulation.

It is observed in the MD simulations of an LCO together with the protein fibril that there is an attraction between the two, with the LCO spending most of the simulation in close proximity to the surface of the protein. It is of interest to see whether this has an effect on the planarity of the LCOs and to test this, three simulations were performed for the p-FTAA chromophore. The first was for the LCO free in water, the second adds a graphene sheet, representing an extreme case, and the third instead adds the protein. The resulting planarity distributions are shown in Figure 9.3. It is evident that graphene has a significant effect on the LCO, causing a large shift of the planarity towards flatter conformations. The simulation with the protein, on the other hand, shows no perceivable shift at all. This indicates that the planarization 
does not occur simply because of the surface offered by the protein, but due to an actual binding pocket in which the LCO is restricted.

\section{Aggregation}

As mentioned in the previous section, the LCOs stay in close proximity to the surface of the protein in the MD simulations. It was hypothesized that this may promote aggregation among the molecules, something which is not observed for LCOs free in water. Such aggregation may in turn affect the spectral properties, providing an explanation for the experimentally observed shifts. This was tested by studying systems containing between one and three p-FTAA molecules with protonated carboxylate groups. The protonated version of p-FTAA shows a strong tendency for aggregation, making it an ideal test case.

Using the method of conformational averaging based on MD sampling, the calculations for the two- and three-LCO systems quickly become very challenging. With multiple LCOs, the conformational complexity of the system increases significantly, and with it the required number of samples needed for an accurate description. Furthermore, the size of the systems for which the spectrum calculation are performed doubles or triples, adding additional resource requirements. It is clear that such calculations are currently not feasible using the high-quality methods that have previously been employed for the single-LCO calculations. Instead, the semi-empirical ZINDO method ${ }^{75}$ was used, in which the core electrons of the system are replaced by an effective potential and all two-electron, non-Coulombic integrals involving two centres are neglected, replaced by parameters based on other calculations or experimental data. ${ }^{27}$ With this method, it is possible to increase both the system size as well as the number of snapshots for which spectra are calculated.

Figure 9.4 shows the calculated spectrum for a single protonated p-FTAA as well those for aggregates of two and three molecules, based on 2400 structural snapshots. While the spectra show slight variations, no major shifts of the main peak are observed. To ensure that this is not simply due to a lack of accuracy in the ZINDO method, a few test calculations were performed to verify the result. The spectra for 10 snapshots containing aggregates of two p-FTAA molecules were calculated using time-dependent DFT at the CAM-B3LYP/aug-cc-pVDZ level of theory and were then compared to the spectra for the individual p-FTAA molecules extracted from the aggregate structures. It was found that the aggregate spectra were almost identical to the sum of the spectra for the individual molecules, indicating that the presence of additional molecules has little effect on the absorption spectrum. It seems instead that the small differences seen in Figure 9.4 are due to slight structural changes. Aggre-

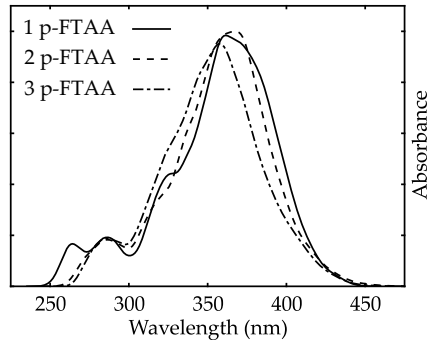

FIGURE 9.4: Averaged absorption spectra for a single protonated $p$-FTAA as well as for aggregates of two and three molecules, calculated using ZINDO based on 2400 structural samples. 
gates of two p-FTAA molecules form an orderly stack, which causes a slight planarization in them, resulting in a small redshift of the spectrum. Aggregates of three p-FTAA molecules, on the other hand, form more clump-like structures, which results in a slight decrease in planarity and a blueshift of the spectrum. These effects are small, however, and aggregation, if it even occurs on the protein, does not have a significant influence on the spectral properties of the LCOs.

\section{Polarization}

While the primary focus has been on the influence of the protein through conformational change in the LCOs, it is also possible that going from an aqueous environment to that of the protein will have effect on the electronic distribution of the LCO, altering its spectral properties. To investigate this, absorption spectra were calculated for p-HTAA using PCM descriptions of water and protein environments. This is a somewhat dubious model already for water, as the discrete nature of the water molecules appears to be important for the description the LCOs, and even more so for the protein, which can hardly be described as homogeneous. However, it does allow direct comparisons to be made between different environments for the same solute structure, eliminating any conformational changes they would cause. A more thorough investigation of this issue would require QM/MM calculations to be performed for a large number of samples in both environments, but for this small study a PCM description will suffice.

Absorption spectra were calculated for 50 structural snapshots, once for the water environment and once for the protein environment. The relative permittivity of a protein, if such a thing can even be defined, is understandably somewhat vague, varying depending on the type of protein and the position on it. ${ }^{76}$ For these calculations, a value on the low end of the scale was chosen to present an environment as different from water as possible. Thus, for the protein, a relative permittivity of 6 was used, while for water a value of 78.39 was used. For the size of the probe, a large radius of $3.125 \AA$ was chosen somewhat arbitrarily, which can be compared to the much smaller radius of $1.385 \AA$ used for water. The wavelength of the dominant first excited state was compared for each pair of spectra, with the protein calculations showing an average redshift of just $3 \mathrm{~nm}$. This indicates, at least based on these rough calculations, that the direct environmental effects on the spectral properties are small. It is possible that a more in depth investigation would show a larger influence, but for now the conformational explanation for the spectral shifts still appears the most likely. 




\section{B I B L IOG R A P HY}

[1] R. Westlund, E. Malmström, C. Lopes, J. Öhgren, T. Rodgers, Y. Saito, S. Kawata, E. Glimsdal, and M. Lindgren. Efficient nonlinear absorbing platinum(II) acetylide chromophores in solid PMMA matrices. Adv. Func. Mat., 18:1939-1948, 2008.

[2] K. P. R. Nilsson, P. Hammarström, F. Ahlgren, A. Herland, E. A. Schnell, M. Lindgren, G. T. Westermark, and O. Inganäs. Conjugated polyelectrolytes - Conformation-sensitive optical probes for staining and characterization of amyloid deposits. ChemBioChem, 7:1096-1104, 2006.

[3] A. Åslund, C. J. Sigurdson, T. Klingstedt, S. Grathwohl, T. Bolmont, D. L. Dickstein, E. Glimsdal, S. Prokop, M. Lindgren, P. Konradsson, D. M. Holtzman, P. R. Hof, F. L. Heppner, S. Gandy, M. Jucker, A. Aguzzi, P. Hammarström, and K. P. R. Nilsson. Novel pentameric thiophene derivatives for in vitro and in vivo optical imaging of a plethora of protein aggregates in cerebral amyloidoses. ACS Chem. Bio., 4:673-684, 2009.

[4] T. Klingstedt and K. P. R. Nilsson. Luminescent conjugated poly- and oligothiophenes: Optical ligands for spectral assigment of a plethora of protein aggregates. Biochem. Soc. Trans., 40:704-710, 2012.

[5] M. Biancalana and S. Koide. Molecular mechanism of thioflavin-T binding to amyloid fibrils. Biochim. Biophys. Acta, 1804:1405-1412, 2010.

[6] P. Frid, S. V. Anisimov, and N. Popovic. Congo red and protein aggregation in neurodegenerative diseases. Brain Res. Rev., 53: 135-160, 2007.

[7] R. C. González Cano, H. Herrera, J. L. Seguera, J. T. López Navarrete, M. C. Ruiz Delgado, and J. Casado. Conformational control of the electronic properties of an $\alpha-\beta$ terthiophene: Lessons from a precursor towards dendritic hyperbranched oligo- and polythiophenes. ChemPhysChem, 13:3893-3900, 2012.

[8] R. C. González Cano, G. Saini, J. Jacob, J. T. López Navarrete, J. Casado, and M. C. Ruiz Delgado. Interplay of $\alpha, \alpha$-versus $\alpha, \beta$-conjugation in the excited states and charged defects of branched oligothiophenes as models for dendrimeric materials. Chem. Eur. J., 19:17165-17171, 2013.

[9] J. Sjöqvist. Luminescence properties of flexible conjugated dyes, 2012. Lic. thesis. Linköping University, Sweden.

[10] P. Atkins and J. de Paula. Physical chemistry, 9th ed. Oxford University Press, 2010.

[11] J. M. Hollas. Modern spectroscopy, 4th ed. John Wiley \& Sons, Ltd, 2004. 
[12] G. Mackinney. Absorption of light by chlorophyll solutions.

J. Biol. Chem., 140:315-322, 1941.

[13] A. Roggan, M. Friebel, K. Dörschel, A. Hahn, and G. Müller. Optical properties of circulating human blood in the wavelength range 400-2500 nm. J. Biomed. Opt., 4:36-46, 1999.

[14] P. F. Bernath. Spectra of atoms and molecules, 2nd ed. Oxford University Press, 2005.

[15] J. R. Lakowicz. Principles of fluorescence spectroscopy, 3rd ed. Springer US, 2006.

[16] D. Kirkpatrick. The separation of optical brighteners by liquid-solid chromatography. J. Chromatogr. A, 121:153-155, 1976.

[17] P. J. Larkin. Infrared and raman spectroscopy: Principles and spectral interpretation. Elsevier, 2011.

[18] A. W. Jones and L. Andersson. Variability of the blood/breath alcohol ratio in drinking drivers. J. Forensic Sci., 41:916-921, 1996.

[19] M. Planck. Ueber das gesetz der energieverteilung im normalspectrum. Ann. Phys., 309:553-563, 1901.

[20] A. Einstein. Über einen die erzeugung und verwandlung des lichtes betreffenden heuristischen gesichtspunkt. Ann. Phys., 322:132-148, 1905.

[21] E. Schrödinger. An undulatory theory of the mechanics of atoms and molecules. Phys. Rev., 28:1049-1070, 1926.

[22] M. Born and R. Oppenheimer. Zur quantentheorie der molekeln. Ann. Phys., 389:457-484, 1927.

[23] C. R. Jacob and M. Reiher. Spin in density-functional theory. Int. J. Quant. Chem., 112:3661-3684, 2012.

[24] P. Hohenberg and W. Kohn. Inhomogeneous electron gas. Phys. Rev., 136:B864-B871, 1964.

[25] W. Kohn and L. J. Sham. Self-consistent equations including exchange and correlation effects. Phys. Rev., 140:A1133-A1138, 1965.

[26] J. P. Perdew and K. Schmidt. Jacob's ladder of density functional approximations for the exchange-correlation energy. AIP Conf. Proc., 577:1-20, 2001.

[27] F. Jensen. Introduction to computational chemistry, 2nd ed. John Wiley \& Sons Ltd., 2006.

[28] S. H. Vosko, L. Wilk, and M. Nusair. Accurate spin-dependent electron liquid correlation energies for local spin density calculations: A critical analysis. Can. J. Phys., 58:1200-1211, 1980. 
[29] J. P. Perdew and Y. Wang. Accurate and simple analytic representation of the electron-gas correlation energy. Phys. Rev. B, 45:13244-13249, 1992.

[30] A. D. Becke. Density-functional exchange-energy approximation with correct asymptotic behavior. Phys. Rev. A, 38:3098-3100, 1988.

[31] C. Lee, W. Yang, and R. G. Parr. Development of the Colle-Salvetti correlation-energy formula into a functional of the electron density. Phys. Rev. B, 37:785-789, 1988.

[32] S. F. Sousa, P. A. Fernandes, and M. J. Ramos. General performance of density functionals. J. Phys. Chem. A, 111:10439-10452, 2007.

[33] A. D. Becke. Density-functional thermochemistry. IV. A new dynamical correlation functional and implications for exact-exchange mixing. J. Chem. Phys., 104:1040-1046, 1996.

[34] Y. Zhao and D. G. Truhlar. A new local density functional for main-group thermochemistry, transition metal bonding, thermochemical kinetics, and noncovalent interactions. J. Chem. Phys., 125:194101, 2006.

[35] A. D. Becke. A new mixing of Hartree-Fock and local density-functional theories. J. Chem. Phys., 98:1372-1377, 1993.

[36] A. D. Becke. Density-functional thermochemistry. III. The role of exact exchange. J. Chem. Phys., 98:5648-5652, 1993.

[37] P. J. Stephens, F. J. Devlin, C. F. Chabalowski, and M. J. Frisch. Ab Initio calculation of vibrational absorption and circular dichroism spectra using density functional force fields. J. Phys. Chem., 98: 11623-11627, 1994.

[38] M. E. Casida. Time-dependent density-functional theory for molecules and molecular solids. J. Mol. Struc. (THEOCHEM), 914: 3-18, 2009.

[39] T. Yanai, D. P. Tew, and N. C. Handy. A new hybrid exchange-correlation functional using the Coulomb-attenuating method (CAM-B3LYP). Chem. Phys. Lett., 393:51-57, 2004.

[40] S. Grimme. Semiempirical GGA-type density functional constructed with a long-range dispersion correction. J. Comp. Chem., 27:1787-1799, 2006.

[41] J. Olsen and P. Jørgensen. Linear and nonlinear response functions for an exact state and for an MCSCF state. J. Chem. Phys., 82:3235-3264, 1985.

[42] J. Schirmer and A. Dreuw. Critique of the foundations of time-dependent density-functional theory. Phys. Rev. A, 75:022513, 2007. 
[43] P. Norman. A perspective on nonresonant and resonant electronic response theory for time-dependent molecular properties.

Phys. Chem. Chem. Phys., 13:20519-20535, 2011.

[44] T. Helgaker, S. Coriani, P. Jørgensen, K. Kristensen, J. Olsen, and K. Ruud. Recent advances in wave function-based methods of molecular-property calculations. Chem. Rev., 112:543-631, 2012.

[45] B. R. Brooks, R. E. Bruccoleri, B. D. Olafson, D. J. States, S. Swaminathan, and M. Karplus. CHARMM: A program for macromolecular energy, minimization, and dynamics calculations. J. Comp. Chem., 4:187-217, 1983.

[46] N. L. Allinger, Y. H. Yuh, and J. H. Lii. Molecular mechanics. The MM3 force field for hydrocarbons. 1. J. Am. Chem. Soc., 111:8551-8566, 1989.

[47] A. C. T. van Duin, S. Dasgupta, F. Lorant, and W. A. Goddard. ReaxFF: A reactive force field for hydrocarbons. J. Phys. Chem. A, 105: 9396-9409, 2001.

[48] P. M. Morse. Diatomic molecules according to the wave mechanics. II. Vibrational levels. Phys. Rev., 34:57-64, 1929.

[49] J. E. Jones. On the determination of molecular fields. II. From the equation of state of a gas. Proc. R. Soc. Lond. A, 106:463-477, 1924.

[50] R. A. Buckingham. The classical equation of state of gaseous helium, neon and argon. Proc. R. Soc. A, 168:264-283, 1938.

[51] L. Verlet. Computer "experiments" on classical fluids. I. Thermodynamical properties of Lennard-Jones molecules. Phys. Rev., 159:98-103, 1967.

[52] W. C. Swope, H. C. Andersen, P. H. Berens, and K. R. Wilson. A computer simulation method for the calculation of equilibrium constants for the formation of physical clusters of molecules: Application to small water clusters. J. Chem. Phys., 76:637-649, 1982.

[53] H. J. C. Berendsen, J. P. M. Postma, W. F. van Gunsteren, A. DiNola, and J. R. Haak. Molecular dynamics with coupling to an external bath. J. Chem. Phys., 81:3684-3690, 1984.

[54] H. C. Andersen. Molecular dynamics simulations at constant pressure and/or temperature. J. Chem. Phys., 72:2384-2393, 1980.

[55] Shuichi Nosè. A molecular dynamics method for simulations in the canonical ensemble. Mol. Phys., 52:255-268, 1984.

[56] Shuichi Nosè. A unified formulation of the constant temperature molecular dynamics methods. J. Chem. Phys., 81:511-519, 1984.

[57] W. G. Hoover. Canonical dynamics: Equilibrium phase-space distributions. Phys. Rev. A, 31:1695-1697, 1985. 
[58] A. Warshel and M. Karplus. Calculation of ground and excited state potential surfaces of conjugated molecules. I. Formulation and parametrization. J. Am. Chem. Soc., 94:5612-5625, 1972.

[59] A. Warshel and M. Levitt. Theoretical studies of enzymic reactions: Dielectric, electrostatic and steric stabilization of the carbonium ion in the reaction of lysozyme. J. Mol. Biol., 103:227-249, 1976.

[60] M. Levitt and A. Warshel. Computer simulation of protein folding. Nature, 253:694-698, 1975.

[61] T. D. Poulsen, J. Kongsted, A. Osted, P. R. Ogilby, and K. V. Mikkelsen. The combined multiconfigurational self-consistent-field/molecular mechanics wave function approach. J. Chem. Phys., 115:2393-2400, 2001.

[62] H. M. Senn and W. Thiel. QM/MM methods for biomolecular systems. Angew. Chem. Int. Ed., 48:1198-1229, 2009.

[63] P. Ahlström, A. Wallqvist, S. Engström, and B. Jönsson. A molecular dynamics study of polarizable water. Mol. Phys., 68:563-581, 1989.

[64] J. Kongsted, A. Osted, K. V. Mikkelsen, and O. Christiansen. The $\mathrm{QM} / \mathrm{MM}$ approach for wavefunctions, energies and response functions within self-consistent field and coupled cluster theories. Mol. Phys., 100:1813-1828, 2002.

[65] A. Kestutis, K. V. Mikkelsen, and J. Kongsted. Modelling spectroscopic properties of large molecular systems. The combined density functional theory/molecular mechanics approach. J. Comput. Methods Sci. Eng., 7:135-158, 2007.

[66] B. Lee and F. M. Richards. The interpretation of protein structures: Estimation of static accessibility. J. Mol. Biol., 55:379-400, 1971.

[67] A. Shrake and J. A. Rupley. Environment and exposure to solvent of protein atoms. Lysozyme and insulin. J. Mol. Biol., 79:351-371, 1973.

[68] M. L. Connolly. Analytical molecular surface calculation. J. Appl. Cryst., 16:548-558, 1983.

[69] J. Tomasi, B. Mennucci, and R. Cammi. Quantum mechanical continuum solvation models. Chem. Rev., 105:2999-3094, 2005.

[70] A. Klamt and G. Schuurmann. COSMO: A new approach to dielectric screening in solvents with explicit expressions for the screening energy and its gradient. J. Chem. Soc., Perkin Trans. 2, 799-805, 1993.

[71] T. Klingstedt, A. Åslund, R. S. Simon, L. B. G. Johansson, J. J. Mason, S. Nyström, P. Hammarström, and K. P. R. Nilsson. Synthesis of a library of oligothiophenes and their utilization as fluorescent ligands for spectral assigment of protein aggregates. Org. Biomol. Chem., 9: 8356-8370, 2011. 
[72] T. Lührs, C. Ritter, M. Adrian, D. Riek-Loher, B. Bohrmann, H. Döbeli, D. Schubert, and R. Riek. 3D structure of Alzheimer's amyloid- $\beta(1-42)$ fibrils. Proc. Natl. Acad. Sci. U.S.A., 102:17342-17347, 2005.

[73] R. Zhang, X. Hu, H. Khant, S. J. Ludtke, W. Chiu, M. F. Schmid, C. Frieden, and J.-M. Lee. Interprotofilament interactions between Alzheimer's A $\beta 1-42$ peptides in amyloid fibrils revealed by cryoEM. Proc. Natl. Acad. Sci. U.S.A., 106:4653-4658, 2009.

[74] G. M. Morris, R. Huey, W. Lindstrom, M. F. Sanner, R. K. Belew, D. S. Goodsell, and A. J. Olson. AutoDock4 and AutoDockTools4: Automated docking with selective receptor flexibility. J. Comput. Chem., 30:2785-2791, 2009.

[75] J. Ridley and M. Zerner. An intermediate neglect of differential overlap technique for spectroscopy: Pyrrole and the azines. Theor. Chim. Acta, 32:111-134, 1973.

[76] L. Li, C. Li, Z. Zhang, and E. Alexov. On the dielectric "constant" of proteins: Smooth dielectric function for macromolecular modeling and its implementation in DelPhi. J. Chem. Theory Comput., 9:

2126-2136, 2013. 


\section{LIST OF FIGURES}

1.1 Platinum(II) acetylide chromophore . . . . . . . . . . . . . . . . 4

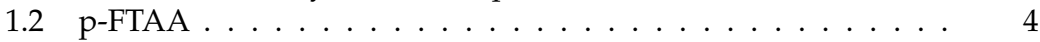

1.3 Fluorescence imaged amyloid aggregates . . . . . . . . . . 4

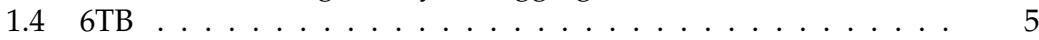

2.1 The electromagnetic spectrum . . . . . . . . . . . . . . 7

2.2 Vibrational broadening . . . . . . . . . . . . . . . 9

2.3 Stokes shift . . . . . . . . . . . . . . . . . . . 10

2.4 The vibrational modes of water . . . . . . . . . . . . . . . 11

2.5 IR spectrum of water . . . . . . . . . . . . . . . . . . . 11

2.6 Raman spectrum of water . . . . . . . . . . . . . 12

3.1 The self-consistent field method . . . . . . . . . . . . . . . . 18

3.2 Jacob's ladder . . . . . . . . . . . . . . . . . . . . . . . 19

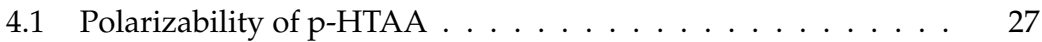

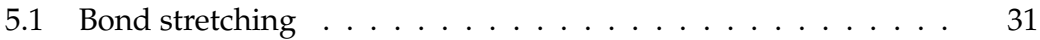

5.2 Methane bond stretching . . . . . . . . . . . . . . 31

5.3 Angle bending . . . . . . . . . . . . . . . . 32

5.4 Water angle bending ................. 32

5.5 Torsion . . . . . . . . . . . . . . . . . 33

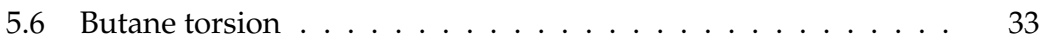

5.7 Out-of-plane bending . . . . . . . . . . . . . . . 34

6.1 Euler integration . . . . . . . . . . . . . . . . . 36

6.2 Verlet integration . . . . . . . . . . . . . . . . . . 36

6.3 Velocity Verlet integration . . . . . . . . . . . 37

7.1 Polarizable water model . . . . . . . . . . . . . . . . . . 42

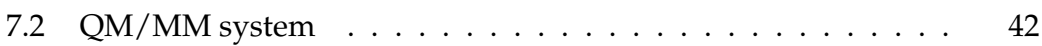

7.3 Cavity generation methods . . . . . . . . . . . . . 45

8.1 Conformational averaging using MD sampling . . . . . . . . . 48

8.2 Fluorescence sampling . . . . . . . . . . . 50

9.1 Luminescent conjugated oligothiophenes . . . . . . . . . . . . . . 51

9.2 Structure levels of amyloid proteins . . . . . . . . . . . . . . . 51

9.3 LCO planarity . . . . . . . . . . . . . . . . . . . . . . 52

9.4 Absorption spectra of LCO aggregates . . . . . . . . . . 53 

Papers 


\section{LIST OF PAPERS AND MY CONTRIBUTIONS}

Paper I Platinum(II) and phosphorus MM3 force field parametrization for chromophore absorption spectra at room temperature

J. Sjöqvist, M. Linares, P. Norman

The Journal of Physical Chemistry A, 114:4981-4987, 2010.

Paper II Molecular dynamics effects on luminescence properties of oligothiophene derivatives: A molecular mechanics-response theory study based on the CHARMM force field and density functional theory

J. Sjöqvist, M. Linares, M. Lindgren, P. Norman

Physical Chemistry Chemical Physics, 13:17532-17542, 2011

Paper III QM/MM-MD simulations of conjugated polyelectrolytes: A study of luminescent conjugated oligothiophenes for use as biophysical probes

J. Sjöqvist, M. Linares, K. V. Mikkelsen, P. Norman

The Journal of Physical Chemistry A, 118:3419-3428, 2014

Paper IV Towards a molecular understanding of the detection of amyloid proteins with flexible conjugated oligothiophenes J. Sjöqvist, J. Maria, R. A. Simon, M. Linares, P. Norman, K. P. R. Nilsson, M. Lindgren Submitted

Paper V A combined MD/QM and experimental exploration of conformational richness in branched oligothiophenes

J. Sjöqvist, R. C. González Cano, J. T. López Navarette, J. Casado, M. C. Ruiz Delgado, M. Linares, P. Norman Submitted

\section{CONTRIBUTIONS TO PAPERS}

Together with Patrick Norman and Mathieu Linares, with additional input from other co-authors, I planned, executed, and analyzed all the theoretical work done in all papers. All calculations were performed by me. For Papers I-III, I wrote the first draft and for Papers IV and V, I wrote the first draft of the theoretical sections. I also participated in the rewriting process and prepared the final manuscripts for submission. 


\section{Papers}

The articles associated with this thesis have been removed for copyright reasons. For more details about these see:

http://urn.kb.se/resolve?urn=urn:nbn:se:liu:diva-109011 